This is a post-peer-review, pre-copyedit version of an article published in Semigroup Forum. The final authenticated version is available online at: https://doi.org/10.1007/s00233-017-9872-7

\title{
REDUCIBILITY OF OPERATOR SEMIGROUPS AND VALUES OF VECTOR STATES
}

\author{
L.W. MARCOUX ${ }^{1}$, H. RADJAVI ${ }^{1}$, AND B.R. YAHAGHI
}

\begin{abstract}
Let $\mathcal{S}$ be a multiplicative semigroup of bounded linear operators on a complex Hilbert space $\mathcal{H}$, and let $\Omega$ be the range of a vector state on $\mathcal{S}$ so that $\Omega=\{\langle S \xi, \xi\rangle: S \in \mathcal{S}\}$ for some fixed unit vector $\xi \in \mathcal{H}$. We study the structure of sets $\Omega$ of cardinality two coming from irreducible semigroups $\mathcal{S}$. This leads us to sufficient conditions for reducibility and, in some cases, for the existence of common fixed points for $\mathcal{S}$. This is made possible by a thorough investigation of the structure of maximal families $\mathcal{F}$ of unit vectors in $\mathcal{H}$ with the property that there exists a fixed constant $\rho \in \mathbb{C}$ for which $\langle x, y\rangle=\rho$ for all distinct pairs $x$ and $y$ in $\mathcal{F}$.
\end{abstract}

\section{INTRODUCTION}

1.1. Let $\mathcal{H}$ be a complex Hilbert space of dimension at least two, and by $\mathcal{B}(\mathcal{H})$ let us denote the algebra of all bounded (i.e. continuous) linear operators on $\mathcal{H}$. A unit vector $\xi \in \mathcal{H}$ defines a vector state $\varphi_{\xi}: \mathcal{B}(\mathcal{H}) \rightarrow \mathbb{C}$ via $\varphi_{\xi}(T)=\langle T \xi, \xi\rangle$ for all $T \in \mathcal{B}(\mathcal{H})$.

Let $\mathcal{S} \subseteq \mathcal{B}(\mathcal{H})$ be a non-empty multiplicative semigroup. In the case where $\mathcal{S}$ is irreducible (that is, where $\mathcal{S}$ admits no non-trivial closed invariant subspaces), it is well known and simple to show (see Lemma 3.2 below) that $\Omega_{\tilde{\zeta}}:=\{\langle S \xi, \xi\rangle: S \in \mathcal{S}\}$ must contain at least two elements. We refer to $\Omega_{\xi}$ as an admissible set for $\mathcal{S}$, and to $\xi$ as an admissible vector corresponding to $\Omega_{\tilde{\xi}}$. It can happen, however, that $\Omega_{\xi}$ will consist of precisely two elements, and in [3], in conjunction with M. Omladič and A.I. Popov, the authors investigated some of the consequences of the existence of admissible sets of cardinality two for an irreducible semigroup $\mathcal{S}$ upon the structure of the semigroup (and the nature of $\Omega_{\xi}$ itself). Almost all of the analysis conducted in that paper was concentrated upon the case where the Hilbert space $\mathcal{H}$ was finite-dimensional. Amongst other things, it was shown in [3] that if $\operatorname{dim} \mathcal{H}=n \geq 2$, and if $\mathcal{S}=\mathcal{S}^{*} \subseteq \mathcal{B}(\mathcal{H}) \simeq \mathbb{M}_{n}(\mathbb{C})$ is a selfadjoint, irreducible semigroup with admissible set $\Omega=\left\{\omega_{1}, \omega_{2}\right\}$, then either every element of $\mathcal{S}$ is invertible and $\mathcal{S}$ is a group, in which case $\Omega=\left\{1,-\frac{1}{n}\right\}$, or $\mathcal{S}$ is unitarily equivalent to $\left\{E_{i j}: 1 \leq i, j \leq n\right\} \cup\{0\}$ and $\Omega=\left\{0, \frac{1}{p}\right\}$ for some integer $1 \leq p \leq n$. Here, $E_{i, j}=e_{i} \otimes e_{j}^{*}$ refers to the standard $(i, j)$-matrix unit in $\mathbb{M}_{n}(\mathbb{C})$ relative to the standard orthonormal basis for $\mathbb{C}^{n}$.

1.2. In this paper we consider semigroups of operators on a complex Hilbert space of arbitrary dimension. For selfadjoint semigroups, and in particular for groups of unitary operators, we find all possible sets $\Omega$ of cardinality two that can occur as an admissible set for irreducible such semigroups. Viewed contrapositively, our results give sufficient

2010 Mathematics Subject Classification. Primary: 46L30. Secondary: 47D03.

Key words and phrases. Irreducible operator semigroups, ranges of vector states, selfadjoint semigroups, equiangular.

${ }^{1}$ Research supported in part by NSERC (Canada).

June 7, 2017. 
conditions for reducibility in terms of the existence of two-element admissible sets $\Omega$. In some cases we are able to prove the existence of a common fixed point for any semigroup that has $\Omega$ as the range of a vector state which acts upon it.

1.3. There is an interesting connection between the question of identifying those irreducible semigroups for which an admissible set of cardinality two exists, and the question of determining families $\Theta$ of unit vectors in $\mathcal{H}$ which have a common inner product: that is, families $\Theta$ for which $\{\langle x, y\rangle: x \neq y \in \Theta\}$ consists of a singleton set.

Indeed, suppose that $\mathcal{S}=\mathcal{S}^{*} \subseteq \mathcal{B}(\mathcal{H})$ is a unital semigroup, i.e. that $I \in \mathcal{B}(\mathcal{H})$ lies in the semigroup $\mathcal{S}$, and that $\Omega=\{\rho, 1\}$ is an admissible set with corresponding admissible vector $\xi$. Extend $\{\xi\}$ to an orthonormal basis $\left\{\xi, e_{\lambda}: \lambda \in \Lambda\right\}$ for $\mathcal{H}$, and let $\Theta=\mathcal{S} \xi:=$ $\{S \xi: S \in \mathcal{S}\}$. If we further suppose that $S \in \mathcal{S}$ implies that $\|S\| \leq 1$ (as we shall see below - see Proposition 3.3 - this assumption is in fact superfluous), then for $S_{1}, S_{2} \in \mathcal{S}$ we have

$$
\left\langle S_{1} \xi, S_{2} \xi\right\rangle=\left\langle S_{2}^{*} S_{1} \xi, \xi\right\rangle \in \Omega,
$$

and the fact that $\left\|S_{1} \xi\right\|,\left\|S_{2} \xi\right\| \leq 1$ shows that $\left\langle S_{1} \xi, S_{2} \xi\right\rangle=1$ implies that $S_{1} \xi=S_{2} \xi$. That is, if $S_{1} \xi \neq S_{2} \xi \in \Theta$, then

$$
\left\langle S_{1} \xi, S_{2} \xi\right\rangle=\rho
$$

is constant.

In Section 2 below, we provide descriptions of those $\rho \in \mathbb{C}$ and of those maximal families $\Theta \subseteq \mathcal{H}$ of unit vectors for which $x \neq y \in \Theta$ implies $\langle x, y\rangle=\rho$. In particular, we shall see that any $0<\rho<1$ gives rise to infinite sets $\Theta$ with this property, while $\rho<0$ implies that the family $\Theta$ must be finite.

1.4. This second problem is related to a famous conjecture known as Zauner's conjecture [5], that for every $n \geq 2$, there exist a collection of $n^{2}$ equiangluar lines, or equivalently, that there exist vectors $\left\{v_{1}, v_{2}, \ldots, v_{n^{2}}\right\} \in \mathbb{C}^{n}$ such that

$$
\left|\left\langle v_{i}, v_{j}\right\rangle\right|=\left\{\begin{array}{ll}
1 & \text { if } i=j \\
\frac{1}{\sqrt{n+1}} & \text { if } i \neq j
\end{array} .\right.
$$

For recent results and references, we refer the reader to [2].

The obvious difference between the two investigations is that in our consideration of inner products, we do not allow for absolute values, in which case it can be shown that the maximum size of a set $\Theta \subseteq \mathbb{C}^{n}$ of unit vectors with the property that $x \neq y \in \Theta$ implies $\langle x, y\rangle=\rho$ is $n+1$, and this occurs precisely when $\rho=-\frac{1}{n}$.

\section{FAMILIES OF UNIT VECTORS WITH CONSTANT INNER PRODUCT}

2.1. We begin our investigations by determining the structure of maximal subsets of a Hilbert space $\mathcal{H}$ for which the inner product of any two distinct members is a fixed constant. We remark that the results of this section hold for real Hilbert spaces (and even quaternionic Hilbert spaces), though we shall focus our attention on the complex case.

2.2. Definition. Let $\mathcal{H}$ be a non-zero, complex Hilbert space, and let $\rho \in \mathbb{C}$. A non-empty family $\Theta \subseteq \mathcal{H}$ of unit vectors is said to have the common inner product of $\rho$ property (the CIP- $\rho$ property) if for all $x \neq y \in \Theta$, we have

$$
\langle x, y\rangle=\rho .
$$


2.3. A few simple remarks are in order:

- The condition is vacuous in the case where $\Theta$ has only one vector; that is, for any unit vector $x \in \mathcal{H}$, the set $\Theta:=\{x\}$ has the CIP- $\rho$ property. For this reason, henceforth, we shall only consider the case where $|\Theta| \geq 2$.

- Since vectors in $\Theta$ are assumed to have norm one, it is a trivial consequence of the Cauchy-Schwarz Inequality that $\Theta$ is empty unless $|\rho| \leq 1$.

- With $|\Theta| \geq 2$, the case $\rho=1$ is impossible. If $x \neq y \in \Theta$ have norm one and $\langle x, y\rangle=1$, then $x=y$ by the Cauchy-Schwarz Inequality. This is a contradiction.

- If $x \neq y \in \Theta$, then $\rho=\langle y, x\rangle=\overline{\langle x, y\rangle}=\bar{\rho}$, whence $\rho \in \mathbb{R}$. That is, $-1 \leq \rho<1$.

Let us dispense with the degenerate cases, namely $\rho=0$ and $\rho=-1$.

(a) The case where $\rho=0$ simply means that $\Theta$ is an orthonormal set in $\mathcal{H}$. Such sets always exist.

(c) Suppose that $\rho=-1$ and fix a vector $x_{0} \in \Theta$. If $y \neq x_{0} \in \Theta$, then $\left\|x_{0}\right\|=$ $1=\|y\|$ and $\left\langle x_{0}, y\right\rangle=-1$ implies that $y=-x_{0}$. In particular, this shows that $\Theta=\left\{x_{0},-x_{0}\right\}$. Conversely, if $x_{0} \in \mathcal{H}$ is any unit vector, then $\Theta:=\left\{x_{0},-x_{0}\right\}$ is a maximal family of unit vectors with the CIP- $(-1)$ property.

2.4. We are left with two cases to consider, namely: $0<\rho<1$, and $-1<\rho<0$. We shall see that these cases behave somewhat differently. More precisely, we will see that, given $0<\rho<1$, maximal families of unit vectors with the CIP- $\rho$ property can always be constructed with the same cardinality as the dimension of the underlying Hilbert space whereas, when $-1<\rho<0$, maximal families of unit vectors with the CIP- $\rho$ are necessarily finite with at most $\left\lfloor\frac{-1}{\rho}\right\rfloor+1$ elements where, for $x \in \mathbb{R},\lfloor x\rfloor=\max \{n \in \mathbb{Z}: n \leq x\}$.

\section{CASE ONE: $0<\rho<1$.}

2.5. Given $0<\rho<1$, we define sequences $\left(r_{n}\right)_{n=1}^{\infty}$ and $\left(s_{n}\right)_{n=1}^{\infty}$, which we call the standard weight sequences associated to $\rho$, as follows

$$
r_{n}=\frac{\rho}{1+(n-1) \rho}, s_{n}=\sqrt{1-r_{n}^{2}},(n \in \mathbb{N})
$$

It is easily checked that $r_{1}=\rho, \lim _{n} r_{n}=0, r_{n+1}=\frac{r_{n}}{1+r_{n}},\left(1+r_{n}\right)\left(1-r_{n+1}\right)=1$, and that $s_{1}^{2} \cdots s_{n}^{2}=\left(1-r_{1}\right)\left(1+r_{n}\right)$ for all $n \in \mathbb{N}$.

Suppose that $N \geq 2$ is an integer and that $\mathcal{E}_{N}:=\left\{e_{1}, e_{2}, \ldots, e_{N}\right\}$ is an orthonormal family of vectors in $\mathcal{H}$.

Define the vectors $x_{n}\left(:=x_{n}[\rho]\right), 1 \leq n \leq N$ as follows:

$$
\begin{aligned}
x_{1} & =e_{1}, \\
x_{2} & =r_{1} e_{1}+s_{1} e_{2}, \\
\vdots & \\
x_{n} & =r_{1} e_{1}+s_{1} r_{2} e_{2}+s_{1} s_{2} r_{3} e_{3}+\cdots+s_{1} s_{2} s_{3} \cdots s_{n-2} r_{n-1} e_{n-1}+s_{1} s_{2} s_{3} \cdots s_{n-1} e_{n} .
\end{aligned}
$$

We then set $\Gamma\left(\mathcal{E}_{N}, \rho\right)=\left\{x_{n}\right\}_{n=1}^{N}$.

If $\mathcal{E}_{\infty}=\left\{e_{n}\right\}_{n=1}^{\infty}$ is a denumerably infinite orthonormal family of vectors in $\mathcal{H}$, then $x_{n}$ is defined for all $n \geq 1$, and we set $\Gamma\left(\mathcal{E}_{\infty}, \rho\right)=\left\{x_{n}\right\}_{n=1}^{\infty}$.

We refer to $\Gamma\left(\mathcal{E}_{N}, \rho\right)$ (resp. $\left.\Gamma\left(\mathcal{E}_{\infty}, \rho\right)\right)$ as the standard vector sequences (the value of $\rho$ is understood to be fixed) associated to $\mathcal{E}_{N}$ (resp. $\mathcal{E}_{\infty}$ ). 
Our first lemma establishes the basic properties of the standard vector sequence associated to $\mathcal{E}$ and $\rho$, where $\mathcal{E}$ is a given family of orthonormal vectors in $\mathcal{H}$.

2.6. Lemma. Let $\mathcal{H}$ be a Hilbert space and $0<\rho<1$.

(a) Suppose that $N \geq 1$ is an integer and that $\mathcal{E}_{N}=\left(e_{n}\right)_{n=1}^{N}$ a finite orthonormal sequence of vectors in $\mathcal{H}$. If $\Gamma\left(\mathcal{E}_{N}, \rho\right)=\left(x_{n}\right)_{n=1}^{N}$ is the standard vector sequence associated to $\mathcal{E}_{N}$, then $\left\langle x_{i}, x_{j}\right\rangle=\rho+\delta_{i, j}(1-\rho)$ for all $1 \leq i, j \leq N$, where $\delta_{i, j}$ denotes the Kronecker delta function.

(b) If $\mathcal{E}_{\infty}=\left\{e_{n}\right\}_{n=1}^{\infty}$ an orthonormal sequence of vectors in $\mathcal{H}$ and $\Gamma\left(\mathcal{E}_{\infty}, \rho\right)=\left(x_{n}\right)_{n=1}^{\infty}$ is the standard vector sequence associated to $\mathcal{E}_{\infty}$, then $\left\langle x_{i}, x_{j}\right\rangle=\rho+\delta_{i, j}(1-\rho)$ for all $i, j \in \mathbb{N}$.

That is to say, both $\Gamma\left(\mathcal{E}_{N}, \rho\right)$ and $\Gamma\left(\mathcal{E}_{\infty}, \rho\right)$ have the $\mathrm{CIP}-\rho$ property.

Proof. Clearly, it suffices to prove (a). It is easily verified that

$$
\begin{aligned}
& \left\langle x_{1}, x_{1}\right\rangle=1, \\
& \left\langle x_{2}, x_{2}\right\rangle=r_{1}^{2}+s_{1}^{2}=1, \\
& \quad \vdots \\
& \left\langle x_{n}, x_{n}\right\rangle=r_{1}^{2}+s_{1}^{2} r_{2}^{2}+\cdots+s_{1}^{2} s_{2}^{2} \cdots s_{n-1}^{2}=1,
\end{aligned}
$$

where $3 \leq n \leq N$. Also, for $i, j \in \mathbb{N}$ with $1 \leq i<j \leq N$, we have

$$
\begin{aligned}
\left\langle x_{i}, x_{j}\right\rangle & =\left\langle x_{i}, x_{i+1}\right\rangle, \\
& =r_{1}^{2}+s_{1}^{2} r_{2}^{2}+\cdots+s_{1}^{2} \cdots s_{i-2}^{2} r_{i-1}^{2}+s_{1}^{2} \cdots s_{i-1}^{2} r_{i}, \\
& =r_{1}^{2}+s_{1}^{2} r_{2}^{2}+\cdots+s_{1}^{2} \cdots s_{i-2}^{2}\left(1-s_{i-1}^{2}\right)+s_{1}^{2} \cdots s_{i-1}^{2} r_{i}, \\
& =\left(r_{1}^{2}+s_{1}^{2} r_{2}^{2}+\cdots+s_{1}^{2} \cdots s_{i-3}^{2} r_{i-2}^{2}+s_{1}^{2} \cdots s_{i-2}^{2}\right)-s_{1}^{2} \cdots s_{i-1}^{2}\left(1-r_{i}\right), \\
& =1-\left(1-r_{1}\right)\left(1+r_{i-1}\right)\left(1-r_{i}\right), \\
& =1-\left(1-r_{1}\right) \\
& =r_{1}=\rho .
\end{aligned}
$$

2.7. Remark. It is worth noting that the Gram-Schmidt process applied to the sequence $\Gamma\left(\mathcal{E}_{N}, \rho\right)=\left\{x_{n}\right\}_{n=1}^{N}$ above yields the orthonormal sequence $\mathcal{E}_{N}=\left(e_{n}\right)_{n=1}^{N}$, and hence $\Gamma\left(\mathcal{E}_{N}, \rho\right)$ is linearly independent and span $\Gamma\left(\mathcal{E}_{N}, \rho\right)=$ span $\mathcal{E}$. In view of the equations defining " $x_{i}$ "s in term of " $e_{i}$ "s $(1 \leq i \leq N)$, the proof is a matter of straightforward calculations (using induction), which is omitted for the sake of brevity.

Similarly, the Gram-Schmidt process applied to the sequence $\Gamma\left(\mathcal{E}_{\infty}, \rho\right)=\left(x_{n}\right)_{n=1}^{\infty}$ returns the orthonormal sequence $\mathcal{E}_{\infty}=\left\{e_{n}\right\}_{n=1}^{\infty}$, and hence $\Gamma\left(\mathcal{E}_{\infty}, \rho\right)$ is linearly independent and $\overline{\operatorname{span}} \Gamma\left(\mathcal{E}_{\infty}, \rho\right)=\overline{\operatorname{span}} \mathcal{E}$.

2.8. Lemma. Let $\mathcal{H}$ be a Hilbert space, and $y \in \mathcal{H}$ be a unit vector.

(a) Let $N \geq 1$ be an integer and suppose that $\mathcal{E}_{N}=\left\{e_{n}\right\}_{n=1}^{N}$ is an orthonormal subset of $\mathcal{H}$. Let $\Gamma\left(\mathcal{E}_{N}, \rho\right)=\left(x_{n}\right)_{n=1}^{N}$ be the standard vector sequence associated to $\mathcal{E}_{N}$. Then $\left\langle y, x_{n}\right\rangle=\rho$ for all $1 \leq n \leq N$ if and only if $\left\langle y, e_{1}\right\rangle=r_{1}=\rho$ and $\left\langle y, e_{n}\right\rangle=s_{1} \cdots s_{n-1} r_{n}$ for all $1<n \leq N$. Moreover, if we let $y_{N}=\sum_{n=1}^{N}\left\langle y, e_{n}\right\rangle e_{n}$, then $\left\|y_{N}\right\|^{2}=1-s_{1}^{2} \cdots s_{N}^{2}=$ $1-\left(1-r_{1}\right)\left(1+r_{N}\right)$. 
(b) Suppose that $\mathcal{E}_{\infty}=\left\{e_{n}\right\}_{n=1}^{\infty}$ is an orthonormal subset of $\mathcal{H}$. If $\Gamma\left(\mathcal{E}_{\infty}, \rho\right)=\left(x_{n}\right)_{n=1}^{\infty}$, then $\left\langle y, x_{n}\right\rangle=\rho$ for all $n \geq 1$ if and only if $\left\langle y, e_{1}\right\rangle=r_{1}=\rho$ and $\left\langle y, e_{n}\right\rangle=s_{1} \cdots s_{n-1} r_{n}$ for all $n>1$. Moreover, if we let $y_{\infty}=\sum_{n=1}^{\infty}\left\langle y, e_{n}\right\rangle e_{n}$, then $\left\|y_{\infty}\right\|^{2}=\lim _{N}\left\|y_{N}\right\|^{2}=r_{1}=\rho$.

Proof. We prove (a). Our proof can be adjusted to easily establish (b). The assertion is easily checked for $N=1$. Fix $2 \leq N \in \mathbb{N}$. We prove the assertion by induction on $n \leq N$. To prove the "only if" implication, suppose we have $\left\langle y, x_{n}\right\rangle=\rho$ for all $1 \leq n \leq N$. We need to show that $\left\langle y, e_{1}\right\rangle=r_{1}$ and $\left\langle y, e_{n}\right\rangle=s_{1} \cdots s_{n-1} r_{n}$ for all $1<n \leq N$. The assertion trivially holds for $n=1$. Suppose the assertion holds for $n<N$. We prove the assertion for $n+1 \leq N$. Write $y=\sum_{i=1}^{N} c_{i} e_{i}+y_{\perp}$, where $y_{\perp} \in \mathcal{H} \ominus \operatorname{span} \mathcal{E}_{N}$. By the induction hypothesis, $c_{1}=\left\langle y, e_{1}\right\rangle=r_{1}$ and $c_{i}=\left\langle y, e_{i}\right\rangle=s_{1} \cdots s_{i-1} r_{i}$ for $2 \leq i \leq n$. We need to show that $c_{n+1}=\left\langle y, e_{n+1}\right\rangle=s_{1} \cdots s_{n} r_{n+1}$. To this end, we can write

$$
\begin{aligned}
r_{1}=\left\langle y, x_{n+1}\right\rangle & =r_{1}^{2}+s_{1}^{2} r_{2}^{2}+\cdots+s_{1}^{2} s_{2}^{2} \cdots s_{n-1}^{2} r_{n}^{2}+s_{1} s_{2} \cdots s_{n} c_{n+1}, \\
& =r_{1}^{2}+s_{1}^{2} r_{2}^{2}+\cdots+s_{1}^{2} s_{2}^{2} \cdots s_{n-1}^{2}\left(1-s_{n}^{2}\right)+s_{1} s_{2} \cdots s_{n} c_{n+1}, \\
& =\left(r_{1}^{2}+s_{1}^{2} r_{2}^{2}+\cdots+s_{1}^{2} s_{2}^{2} \cdots s_{n-1}^{2}\right)-s_{1}^{2} s_{2}^{2} \cdots s_{n-1}^{2} s_{n}^{2}+s_{1} s_{2} \cdots s_{n} c_{n+1}, \\
& =1-\left(1-r_{1}\right)\left(1+r_{n}\right)+s_{1} s_{2} \cdots s_{n} c_{n+1} .
\end{aligned}
$$

This yields

$$
\begin{aligned}
c_{n+1} & =\frac{1-r_{1}}{s_{1} s_{2} \cdots s_{n}} r_{n} \\
& =\frac{1-r_{1}}{s_{1}^{2} s_{2}^{2} \cdots s_{n}^{2}} s_{1} s_{2} \cdots s_{n} r_{n}, \\
& =\frac{1-r_{1}}{\left(1-r_{1}\right)\left(1+r_{n}\right)} s_{1} s_{2} \cdots s_{n} r_{n}, \\
& =s_{1} s_{2} \cdots s_{n} \frac{r_{n}}{1+r_{n}} \\
& =s_{1} s_{2} \cdots s_{n} r_{n+1}
\end{aligned}
$$

as desired.

Next, to prove the "if" implication, suppose $\left\langle y, e_{1}\right\rangle=r_{1}$ and $\left\langle y, e_{n}\right\rangle=s_{1} \cdots s_{n-1} r_{n}$ for all $1<n \leq N$. We need to show that $\left\langle y, x_{n}\right\rangle=\rho$ for all $1 \leq n \leq N$. Again this trivially holds for $n=1$. Assuming that the assertion holds for $n<N$, we prove it for $n+1 \leq N$. We can write

$$
\begin{aligned}
\left\langle y, x_{n+1}\right\rangle & =r_{1}^{2}+s_{1}^{2} r_{2}^{2}+\cdots+s_{1}^{2} \cdots s_{n-2}^{2} r_{n-1}^{2}+s_{1}^{2} \cdots s_{n-1}^{2} r_{n} \\
& =r_{1}^{2}+s_{1}^{2} r_{2}^{2}+\cdots+s_{1}^{2} \cdots s_{n-2}^{2}\left(1-s_{n-1}^{2}\right)+s_{1}^{2} \cdots s_{n-1}^{2} r_{n} \\
& =\left(r_{1}^{2}+s_{1}^{2} r_{2}^{2}+\cdots+s_{1}^{2} \cdots s_{n-3}^{2} r_{n-2}^{2}+s_{1}^{2} \cdots s_{n-2}^{2}\right)-s_{1}^{2} \cdots s_{n-1}^{2}\left(1-r_{n}\right) \\
& =1-\left(1-r_{1}\right)\left(1+r_{n-1}\right)\left(1-r_{n}\right), \\
& =1-\left(1-r_{1}\right) \times 1 \\
& =r_{1}=\rho,
\end{aligned}
$$


proving the assertion. Finally, we have that

$$
\begin{aligned}
\left\langle y_{N}, y_{N}\right\rangle & =r_{1}^{2}+s_{1}^{2} r_{2}^{2}+\cdots+s_{1}^{2} \cdots s_{N-2}^{2} r_{N-1}^{2}+s_{1}^{2}+\cdots s_{N-1}^{2} r_{N}^{2}, \\
& =r_{1}^{2}+s_{1}^{2} r_{2}^{2}+\cdots+s_{1}^{2} \cdots s_{N-2}^{2}\left(1-s_{N-1}^{2}\right)+s_{1}^{2}+\cdots s_{N-1}^{2} r_{N}^{2}, \\
& =\left(r_{1}^{2}+s_{1}^{2} r_{2}^{2}+\cdots+s_{1}^{2} \cdots s_{N-2}^{2}\right)-s_{1}^{2} \cdots s_{N-1}^{2}+s_{1}^{2} \cdots s_{N-1}^{2} r_{N}^{2}, \\
& =1-s_{1}^{2} \cdots s_{N-1}^{2}\left(1-r_{N}^{2}\right), \\
& =1-s_{1}^{2} \cdots s_{N-1}^{2} s_{N}^{2} \\
& =1-\left(1-r_{1}\right)\left(1+r_{N}\right) .
\end{aligned}
$$

This completes the proof.

It is worth noting that the boundedness of the sequence $\left(x_{n}\right)_{n=1}^{\infty}$ combined with the fact that the $k^{\text {th }}$-coordinate of $x_{n}$ converges to the $k^{t h}$-coordinate of $y_{\infty}$ implies that $y_{\infty}$ is a weak-limit of the sequence $\left(x_{n}\right)_{n=1}^{\infty}$.

2.9. Proposition. Let $\mathcal{H}$ be a Hilbert space.

(a) If $1<\operatorname{dim} \mathcal{H}=N<\infty$ and $\mathcal{E}_{N}=\left\{e_{n}\right\}_{n=1}^{N}$ is an orthonormal basis for $\mathcal{H}$, then $\Gamma\left(\mathcal{E}_{N}, \rho\right)$ is a maximal family of unit vectors with the CIP $-\rho$ property.

(b) If $\operatorname{dim} \mathcal{H}=\aleph_{0}$, and if $\mathcal{E}_{\infty}=\left\{e_{n}\right\}_{n=1}^{\infty}$ is an orthonormal basis for $\mathcal{H}$, then $\Gamma\left(\mathcal{E}_{\infty}, \rho\right)$ is a maximal family of unit vectors in $\mathcal{H}$ with the $\mathrm{CIP}-\rho$ property.

\section{Proof.}

(a) We proceed by contradiction. Suppose that $y \in \mathcal{H}$ is a unit vector and that $\left\langle y, x_{n}\right\rangle=$ $\rho$ for all $1 \leq n \leq N$. It thus follows from Lemma 2.8(a) that $\left\langle y, e_{1}\right\rangle=r_{1}$ and $\left\langle y, e_{n}\right\rangle=s_{1} \cdots s_{n-1} r_{n}$ for all $1<n \leq N$. Thus we can write

$$
\begin{aligned}
1=\langle y, y\rangle & =r_{1}^{2}+s_{1}^{2} r_{2}^{2}+\cdots+s_{1}^{2} \cdots s_{N-2}^{2} r_{N-1}^{2}+s_{1}^{2}+\cdots s_{N-1}^{2} r_{N^{\prime}}^{2} \\
& =r_{1}^{2}+s_{1}^{2} r_{2}^{2}+\cdots+s_{1}^{2} \cdots s_{N-2}^{2}\left(1-s_{N-1}^{2}\right)+s_{1}^{2}+\cdots s_{N-1}^{2} r_{N}^{2} \\
& =\left(r_{1}^{2}+s_{1}^{2} r_{2}^{2}+\cdots+s_{1}^{2} \cdots s_{N-2}^{2}\right)-s_{1}^{2} \cdots s_{N-1}^{2}+s_{1}^{2} \cdots s_{N-1}^{2} r_{N}^{2} \\
& =1-s_{1}^{2} \cdots s_{N-1}^{2}\left(1-r_{N}^{2}\right) \\
& =1-s_{1}^{2} \cdots s_{N-1}^{2} s_{N^{\prime}}^{2} \\
& =1-\left(1-r_{1}\right)\left(1+r_{N}\right)
\end{aligned}
$$

implying that $\left(1-r_{1}\right)\left(1+r_{N}\right)=0$, which is clearly false. This proves the assertion.

(b) Suppose by contradiction that $y \in \mathcal{H}$ is a unit vector and $\left\langle y, x_{n}\right\rangle=\rho$ for all $n \geq 1$.

It then follows from Lemma 2.8(b) that $\left\langle y, e_{1}\right\rangle=r_{1}$ and $\left\langle y, e_{n}\right\rangle=s_{1} \cdots s_{n-1} r_{n}$ for all 
$n>1$. This leads to a contradiction, for we will then obtain

$$
\begin{aligned}
1=\langle y, y\rangle & =\lim _{n}\left(r_{1}^{2}+s_{1}^{2} r_{2}^{2}+\cdots+s_{1}^{2} \cdots s_{n-2}^{2} r_{n-1}^{2}+s_{1}^{2}+\cdots s_{n-1}^{2} r_{n}^{2}\right), \\
& =\lim _{n}\left(r_{1}^{2}+s_{1}^{2} r_{2}^{2}+\cdots+s_{1}^{2} \cdots s_{n-2}^{2}\left(1-s_{n-1}^{2}\right)+s_{1}^{2}+\cdots s_{n-1}^{2} r_{n}^{2}\right), \\
& =\lim _{n}\left(\left(r_{1}^{2}+s_{1}^{2} r_{2}^{2}+\cdots+s_{1}^{2} \cdots s_{n-2}^{2}\right)-s_{1}^{2} \cdots s_{n-1}^{2}+s_{1}^{2} \cdots s_{n-1}^{2} r_{n}^{2}\right), \\
& =\lim _{n}\left(1-s_{1}^{2} \cdots s_{n-1}^{2}\left(1-r_{n}^{2}\right)\right), \\
& =\lim _{n}\left(1-s_{1}^{2} \cdots s_{n-1}^{2} s_{n}^{2}\right), \\
& =\lim _{n}\left(1-\left(1-r_{1}\right)\left(1+r_{n}\right)\right), \\
& =r_{1}=\rho,
\end{aligned}
$$

a contradiction. This completes the proof.

2.10. We are left to consider the case where the underlying Hilbert space $\mathcal{H}$ is not separable.

In this case, suppose that $\mathcal{E}_{\infty}$ is a countable orthonormal set, and extend $\mathcal{E}_{\infty}$ to an orthonormal basis $\mathcal{G}=\mathcal{E}_{\infty} \cup \mathcal{F}$ for $\mathcal{H}$. Write $\mathcal{F}=\left\{f_{\lambda}\right\}_{\lambda \in \Lambda}$.

Let $\Gamma\left(\mathcal{E}_{\infty}, \rho\right)$ be the standard vector sequence associated to $\mathcal{E}_{\infty}$ (and $\rho$ ), and set $y=$ $\sum_{n=1}^{\infty} c_{n} e_{n}$, where $c_{1}=r_{1}$, and $c_{j}=s_{1} s_{2} \cdots s_{j-1} r_{j}, j \geq 2$. Finally, for each $\lambda \in \Lambda$, set

$$
g_{\lambda}=y+\sqrt{1-\rho} f_{\lambda} .
$$

2.11. Proposition. With the above notation, $\Gamma\left(\mathcal{E}_{\infty}, \mathcal{F}, \rho\right):=\Gamma\left(\mathcal{E}_{\infty}, \rho\right) \cup\left\{g_{\lambda}\right\}_{\lambda \in \Lambda}$ is a maximal family of unit vectors in $\mathcal{H}$ with the CIP- $\rho$ property. Furthermore, $\Gamma\left(\mathcal{E}_{\infty}, \mathcal{F}, \rho\right)$ is a linearly independent set whose closed span is $\mathcal{H}$.

Proof. From the proof of Lemma 2.8(b), we see that if $y=\sum_{n=1}^{\infty} c_{n} e_{n}$, then $\|y\|^{2}=\rho$, and thus $y \notin \Gamma\left(\mathcal{E}_{\infty}, \rho\right)$. Moreover, $\langle y, x\rangle=\rho$ for all $x \in \Gamma\left(\mathcal{E}_{\infty}, \rho\right)$. Note further that

$$
\begin{aligned}
\left\langle x, y+\sqrt{1-\rho} f_{\lambda}\right\rangle & =\langle x, y\rangle+\sqrt{1-\rho}\left\langle x, f_{\lambda}\right\rangle, \\
& =\rho+\sqrt{1-\rho}(0), \\
& =\rho, \\
\left\langle y+\sqrt{1-\rho} f_{\lambda}, y+\sqrt{1-\rho} f_{\lambda}\right\rangle & =\langle y, y\rangle+\sqrt{1-\rho}\left(\left\langle y, f_{\lambda}\right\rangle+\left\langle f_{\lambda}, y\right\rangle\right)+(1-\rho)\left\langle f_{\lambda}, f_{\lambda}\right\rangle, \\
& =\rho+\sqrt{1-\rho}(0)+(1-\rho)(1), \\
& =1,
\end{aligned}
$$

and

$$
\begin{aligned}
\left\langle y+\sqrt{1-\rho} f_{\lambda_{1}}, y+\sqrt{1-\rho} f_{\lambda_{2}}\right\rangle & =\langle y, y\rangle+\sqrt{1-\rho}\left(\left\langle y, f_{\lambda_{2}}\right\rangle+\left\langle f_{\lambda_{1}}, y\right\rangle\right)+(1-\rho)\left\langle f_{\lambda_{1}}, f_{\lambda_{2}}\right\rangle, \\
& =\rho+\sqrt{1-\rho}(0)+(1-\rho)(0), \\
& =\rho,
\end{aligned}
$$


for all $x \in \Gamma\left(\mathcal{E}_{\infty}, \rho\right)$ and $\lambda, \lambda_{1}, \lambda_{2} \in \Lambda$ with $\lambda_{1} \neq \lambda_{2}$. This shows that $\Gamma\left(\mathcal{E}_{\infty}, \mathcal{F}, \rho\right)$ is a family of unit vectors in $\mathcal{N}$ for which any two distinct elements have a common inner product of $\rho$.

To show that $\Gamma\left(\mathcal{E}_{\infty}, \mathcal{F}, \rho\right)$ is maximal, suppose $z \in \mathcal{H}$ is a unit vector such that $\langle z, x\rangle=\rho$ for all $x \in \Gamma\left(\mathcal{E}_{\infty}, \mathcal{F}, \rho\right) \backslash\{z\}$. We need to show that $z \in \Gamma\left(\mathcal{E}_{\infty}, \mathcal{F}, \rho\right)$. Suppose by contradiction that $z \notin \Gamma\left(\mathcal{E}_{\infty}, \mathcal{F}, \rho\right)$. In particular, $z \notin \Gamma\left(\mathcal{E}_{\infty}, \rho\right)$ but $\langle z, x\rangle=\rho$ for all $x \in \Gamma\left(\mathcal{E}_{\infty}, \rho\right) \backslash\{z\}$. It thus follows from Lemma 2.8 that $\left\langle z, e_{1}\right\rangle=r_{1}$ and $\left\langle z, e_{n}\right\rangle=s_{1} \cdots s_{n-1} r_{n}$ for all $n>1$. So we can write $z=y+t$, where $t \in \mathcal{H} \ominus \mathcal{M}$. Then again $\left\langle y+t, y+\sqrt{1-\rho} f_{\lambda}\right\rangle=\rho$ for all $\lambda \in \Lambda$, which easily implies $\left\langle t, f_{\lambda}\right\rangle=0$ for all $\lambda \in \Lambda$. This means $t=0$, and hence $z=y$. But once again as we saw in the proof of part (b) of the preceding proposition $1=\langle z, z\rangle=\langle y, y\rangle=\rho$, a contradiction, proving the assertion.

Finally, by Remark 2.7, $e_{n} \in \operatorname{span} \Gamma\left(\mathcal{E}_{\infty}, \rho\right)$ for all $n \in \mathbb{N}$ and so $y=\sum_{n=1}^{\infty} c_{n} e_{n} \in$ $\overline{\operatorname{span}} \Gamma\left(\mathcal{E}_{\infty}, \rho\right)=\mathcal{M}$. In view of the fact that $g_{\lambda}=y+\sqrt{1-r} f_{\lambda}$, this yields that $f_{\lambda} \in$ $\overline{\operatorname{span}} \Gamma\left(\mathcal{E}_{\infty}, \mathcal{F}, \rho\right)$ for all $\lambda \in \Lambda$. Consequently, $\overline{\operatorname{span}} \Gamma\left(\mathcal{E}_{\infty}, \mathcal{F}, \rho\right)=\mathcal{H}$ because $\overline{\operatorname{span}} \Gamma\left(\mathcal{E}_{\infty}, \mathcal{F}, \rho\right)$ contains the orthonormal basis $\mathcal{E} \cup \mathcal{F}$ for $\mathcal{H}$. This completes the proof.

It is worth noting that the fact that $1 \neq \rho=\|y\|^{2}$ clearly implies that $y \notin \Gamma\left(\mathcal{E}_{\infty}, \mathcal{F}, \rho\right)$.

Having produced examples of maximal families of vectors with the CIP- $\rho$ property for $0<\rho<1$, we now prove that any such family is of one of the types exhibited above.

2.12. Theorem. Let $\mathcal{H}$ be a Hilbert space and suppose that $0<\rho<1$. Suppose also that $\Theta \subseteq \mathcal{H}$ is a family of unit vectors such that $x \neq y \in \Theta$ implies that $\langle x, y\rangle=\rho$. Then $\Theta$ is linearly independent, and moreover

(a) If $\mathcal{M}:=\overline{\text { span }} \Theta$ is separable, then there exists an orthonormal basis $\mathcal{E}=\left\{e_{n}\right\}_{n=1}^{N}$ for $\mathcal{M}$ - (here $N \in \mathbb{N} \cup\{\infty\})$ - such that $\Theta=\Gamma(\mathcal{E}, \rho)$.

(b) If $\mathcal{N}:=\overline{\text { span }} \Theta$ is not separable and $\Theta_{0}$ is a countable subset of $\Theta$, then there exist orthonormal bases $\mathcal{E}_{\infty}=\left(e_{n}\right)_{n=1}^{\infty}$ for $\mathcal{M}:=\overline{\text { span }} \Theta_{0}$, and $\mathcal{F}=\left\{f_{\lambda}\right\}_{\lambda \in \Lambda}$ for $\mathcal{N} \ominus \mathcal{M}$ such that $\mathcal{G}=\mathcal{E}_{\infty} \cup \mathcal{F}$ is an orthonormal basis for $\mathcal{N}$ and $\Theta=\Gamma\left(\mathcal{E}_{\infty}, \mathcal{F}, \rho\right)$.

(c) The family $\Theta$ is maximal if and only if $\overline{\operatorname{span}} \Theta=\mathcal{H}$. Moreover, if $\Theta$ is maximal, then $\Theta=\Gamma(\mathcal{E}, \rho),(N \in \mathbb{N} \cup\{\infty\})$ or $\Theta=\Gamma\left(\mathcal{E}_{\infty}, \mathcal{F}, \rho\right)$ depending on whether $\mathcal{H}$ is separable or not, where $\mathcal{E}$ or $\mathcal{E}_{\infty} \cup \mathcal{F}$ are suitable orthonormal bases for $\mathcal{H}$ as described in (a) and (b).

Proof. To see that $\Theta$ is linearly independent, suppose that we are given $n>1,\left\{x_{1}, \ldots, x_{n}\right\} \subseteq$ $\Theta$, and scalars $c_{1}, c_{2}, \ldots, c_{n} \in \mathbb{C}$ so that $c_{1} x_{1}+c_{2} x_{2}+\cdots+c_{n} x_{n}=0$. Then for each $1 \leq k \leq n$, we have

$$
0=\left\langle 0, x_{k}\right\rangle=\left\langle c_{1} x_{1}+c_{2} x_{2}+\cdots+c_{n} x_{n}, x_{k}\right\rangle,
$$

which gives rise to a homogeneous system of $n$ linear equations in $c_{1}, \ldots, c_{n}$ whose coefficient matrix is

$$
R_{n}:=\left[\begin{array}{ccccc}
1 & \rho & \rho & \cdots & \rho \\
\rho & 1 & \rho & \cdots & \rho \\
\vdots & \vdots & & \cdots & \vdots \\
\rho & \rho & \cdots & \rho & 1
\end{array}\right] \in M_{n}(\mathbb{R})
$$

Since $\operatorname{det} R_{n}=(1-\rho)^{n-1}(1+(n-1) \rho) \neq 0$ for all $n \in \mathbb{N}$, we see that $c_{i}=0$ for all $1 \leq i \leq n$. Thus $\left\{x_{1}, \ldots, x_{n}\right\}$ is linearly independent, proving the assertion.

(a) If $\mathcal{M}=\overline{\text { span }} \Theta$ is separable, then observe that $\Theta$ is at most countable, since $x \neq y \in \Theta$ implies that $\|x-y\|^{2}=\|x\|^{2}+\|y\|^{2}-\langle x, y\rangle-\langle y, x\rangle=2-2 \rho>0$. It is an easy and standard exercise to show that a separable Banach space (in this case $\mathcal{M}$ ) can not contain 
an uncountable collection of vectors, each of which is a fixed positive distance away from any other vector in the collection). Hence $\Theta=\left(x_{n}\right)_{n=1}^{N}$ for some $N \in \mathbb{N} \cup\{\infty\}$, and $\langle x, y\rangle=\rho$ for all $x, y \in \Theta$ with $x \neq y$. We prove the assertion for the case $N=\infty$. The assertion for the case $N \in \mathbb{N}$ can be proved in a similar fashion. So let $N=\infty$ and apply the Gram-Schmidt process to $\Theta=\left(x_{n}\right)_{n=1}^{\infty}$, which is linearly independent, to obtain an orthonormal basis $\mathcal{E}_{\infty}=\left(e_{n}\right)_{n=1}^{\infty}$ for the Hilbert space $\mathcal{M}=\overline{\text { span }} \Theta$. We claim that $\Theta=\Gamma\left(\mathcal{E}_{\infty}, \rho\right)$. We need to show that $x_{n}=x_{n}[\rho]$ for all $n \in \mathbb{N}$. Here

$$
\begin{aligned}
& x_{1}[\rho]=e_{1}, \\
& x_{2}[\rho]=r_{1} e_{1}+s_{1} e_{2}, \\
& \vdots \\
& x_{n}[\rho]=r_{1} e_{1}+s_{1} r_{2} e_{2}+s_{1} s_{2} r_{3} e_{3}+\cdots+s_{1} s_{2} s_{3} \cdots s_{n-2} r_{n-1} e_{n-1}+s_{1} s_{2} s_{3} \cdots s_{n-1} e_{n},
\end{aligned}
$$

where $n \geq 3$. We prove this by induction on $n$. That $x_{1}=x_{1}[\rho]=e_{1}$ and $x_{2}=x_{2}[\rho]=$ $r_{1} e_{1}+s_{1} e_{2}$ are relatively straightforward. Assuming that $x_{i}=x_{i}[\rho]$ for all $1 \leq i \leq n$, we prove that $x_{n+1}=x_{n+1}[\rho]$. Write $x_{n+1}=c_{1} e_{1}+\cdots+c_{n} e_{n}+c_{n+1} e_{n+1}$. We prove by induction on $1 \leq i \leq n$ that $\left\langle x_{n+1}, x_{i}\right\rangle=\rho$ implies that $c_{1}=r_{1}$ and $c_{j}=s_{1} \cdots s_{j-1} r_{j}$ whenever $1<j \leq i$. As soon as we establish this, from $\left\langle x_{n+1}, x_{n}\right\rangle=\rho$ and $\left\langle x_{n+1}, x_{n+1}\right\rangle=1$ and the fact that $c_{n+1}>0$ (a consequence of the Gram-Schmidt process), we obtain $c_{1}=$ $r_{1}, c_{i}=s_{1} s_{2} s_{3} \cdots s_{i-1} r_{i}(1<i \leq n)$, and $c_{n+1}=s_{1} s_{2} s_{3} \cdots s_{n}$, and thus we will see that $x_{n+1}=x_{n+1}[\rho]$, as desired. Now, if $i=1$ and $\left\langle x_{n+1}, x_{1}\right\rangle=\rho$, as $x_{1}=e_{1}$, we see that $c_{1}=r_{1}$. Suppose $1 \leq i<n$ and $\left\langle x_{n+1}, x_{i}\right\rangle=\rho$ implies $c_{1}=r_{1}$ and $c_{j}=s_{1} \cdots s_{j-1} r_{j}$ whenever $1<j \leq i$. Suppose $i+1 \leq n$ and $\left\langle x_{n+1}, x_{i+1}\right\rangle=\rho$. By the induction hypothesis $c_{1}=r_{1}$ and $c_{j}=s_{1} \cdots s_{j-1} r_{j}$ whenever $1<j \leq i$ because $\left\langle x_{n+1}, x_{i}\right\rangle=\rho$. But $x_{i+1}=x_{i+1}[\rho]$. Thus we can write

$$
\begin{aligned}
r_{1}=\left\langle x_{n+1}, x_{i+1}\right\rangle & =r_{1}^{2}+s_{1}^{2} r_{2}^{2}+\cdots+s_{1}^{2} s_{2}^{2} \cdots s_{i-1}^{2} r_{i}^{2}+s_{1} s_{2} \cdots s_{i} c_{i+1}, \\
& =r_{1}^{2}+s_{1}^{2} r_{2}^{2}+\cdots+s_{1}^{2} s_{2}^{2} \cdots s_{i-1}^{2}\left(1-s_{i}^{2}\right)+s_{1} s_{2} \cdots s_{i} c_{i+1}, \\
& =\left(r_{1}^{2}+s_{1}^{2} r_{2}^{2}+\cdots+s_{1}^{2} s_{2}^{2} \cdots s_{i-1}^{2}\right)-s_{1}^{2} s_{2}^{2} \cdots s_{i-1}^{2} s_{i}^{2}+s_{1} s_{2} \cdots s_{i} c_{i+1}, \\
& =1-\left(1-r_{1}\right)\left(1+r_{i}\right)+s_{1} s_{2} \cdots s_{i} c_{i+1} .
\end{aligned}
$$

This yields

$$
\begin{aligned}
c_{i+1} & =\frac{1-r_{1}}{s_{1} s_{2} \cdots s_{i}} r_{i}, \\
& =\frac{1-r_{1}}{s_{1}^{2} s_{2}^{2} \cdots s_{i}^{2}} s_{1} s_{2} \cdots s_{i} r_{i}, \\
& =\frac{1-r_{1}}{\left(1-r_{1}\right)\left(1+r_{i}\right)} s_{1} s_{2} \cdots s_{i} r_{i}, \\
& =s_{1} s_{2} \cdots s_{i} \frac{r_{i}}{1+r_{i}}, \\
& =s_{1} s_{2} \cdots s_{i} r_{i+1},
\end{aligned}
$$

which is what we want, proving the assertion.

(b) Note first that $\Theta$, and hence $\Theta_{0}$, is linearly independent. Let $\Theta_{0}=\left(x_{n}\right)_{n=1}^{\infty}$ and $\mathcal{M}=\overline{\text { span }} \Theta_{0}$. Let $\mathcal{E}_{\infty}=\left(e_{n}\right)_{n=1}^{\infty}$ be the orthonormal basis obtained for the Hilbert space $\mathcal{M}=\overline{\text { span }} \Theta_{0}$ by applying the Gram-Schmidt process to $\Theta_{0}=\left(x_{n}\right)_{n=1}^{\infty}$. As we saw in the above $\Theta_{0}=\Gamma\left(\mathcal{E}_{\infty}, \rho\right)$. We claim that $\left(\Theta \backslash \Theta_{0}\right) \cap \mathcal{M}=\varnothing$. Suppose to the contrary that there exists a $y \in\left(\Theta \backslash \Theta_{0}\right) \cap \mathcal{M}$. As $y \in \mathcal{M}$, we have $\left\langle y, f_{\lambda}\right\rangle=0$ for all $\lambda \in \Lambda$. On the other 
hand, from Lemma 2.8(ii), we get that $c_{1}:=\left\langle y, e_{1}\right\rangle=r_{1}$ and $c_{n}:=\left\langle y, e_{n}\right\rangle=s_{1} \cdots s_{n-1} r_{n}$ for all $n>1$. Consequently, $y=\sum_{n=1}^{\infty} c_{n} e_{n}$. But then again as we saw in the proof of Lemma 2.8(b), this yields $1=\langle y, y\rangle=\rho$, which is impossible. Therefore, $\left(\Theta \backslash \Theta_{0}\right) \cap$ $\mathcal{M}=\varnothing$. Set $\Theta \backslash \Theta_{0}=\left(z_{\lambda}\right)_{\lambda \in \Lambda}$. Clearly, given an arbitrary $z_{\lambda} \in \Theta \backslash \Theta_{0}$ with $\lambda \in \Lambda$, in view of Lemma 2.8(b), there is an $f_{\lambda} \in \mathcal{N} \ominus \mathcal{M}$ such that $z_{\lambda}=y+\sqrt{1-\rho} f_{\lambda}$. Since $\Theta \backslash \Theta_{0}=\left(z_{\lambda}\right)_{\lambda \in \Lambda}$ consists of unit vectors and has the common inner product property for $\rho$, we see that $\left(f_{\lambda}\right)_{\lambda \in \Lambda}$ is an orthonormal system of vectors in $\mathcal{N} \ominus \mathcal{M}$. But if $f \in \mathcal{N} \ominus \mathcal{M}$ and $\left\langle f, f_{\lambda}\right\rangle=0$ for all $\lambda \in \Lambda$, then $\langle f, x\rangle=0$ for all $x \in \Theta$, implying that $f=0$ because $\mathcal{N}=\overline{\text { span }} \Theta$. This shows that $\mathcal{F}:=\left(f_{\lambda}\right)_{\lambda \in \Lambda}$ is an orthonormal basis for $\mathcal{N} \ominus \mathcal{M}$. Summing up, we conclude that $\mathcal{G}=\mathcal{E} \cup \mathcal{F}$ is an orthonormal basis for $\mathcal{N}$ and $\Theta=\Gamma\left(\mathcal{E}_{\infty}, \mathcal{F}, \rho\right)$, which is what we want.

(c) The "if" implication is a consequence of (a), (b), and Proposition 2.11. The "only if" implication follows from (a) and (b). To see this, suppose $\Theta$ is maximal but $\overline{\operatorname{span}} \Theta \neq \mathcal{H}$. First, if $\overline{\text { span } \Theta}$ is separable, we see from (a) that there exists an orthonormal basis $\mathcal{E}=$ $\left\{e_{n}\right\}_{n=1}^{N}$ with $N \in \mathbb{N} \cup\{\infty\}$ for $\overline{\text { span }} \Theta$ so that $\Theta=\Gamma(\mathcal{E}, \rho)$ and $\overline{\operatorname{span}} \Theta=\overline{\operatorname{span}} \mathcal{E}$.

Let $\Gamma(\mathcal{E}, \rho)=\left(x_{n}\right)_{n=1}^{N}$ and let $y=\sum_{n=1}^{N} c_{n} e_{n}$ be defined as in paragraph 2.10, so that $c_{1}=r_{1}$ and $c_{j}=s_{1} s_{2} \cdots s_{j-1} r_{j}$ for $j \geq 2$. Choose $f_{0} \in \mathcal{E}^{\perp}$, and define

$$
g_{0}=y+\sqrt{1-\rho} f_{0} .
$$

A simple calculation, identical to that found in Proposition 2.11, shows that $\left\|g_{0}\right\|=1$ and that $\left\langle g_{0}, x_{n}\right\rangle=\rho$ for all $1 \leq \rho \leq N$, contradicting the maximality of $\Theta$. The rest of the assertion evidently follows from (a) and (b). This completes the proof.

\section{CASE Two: $-1<\rho<0$.}

2.13. We now turn our attention to the case where $-1<\rho<0$, an assumption which we shall maintain for the remainder of this section. As previously mentioned, we shall see that, independent of the dimension of the underlying Hilbert space, any family $\Theta$ with the CIP $-\rho$ property must be finite and have at most $\left\lfloor-\frac{1}{\rho}\right\rfloor+1$ elements.

As we did in the case where $0<\rho<1$, we can define the standard weight sequences $\left(r_{i}\right)_{i=1}^{\infty}$ and $\left(s_{i}\right)_{i=1}^{\infty}$, and we can use these to define the coordinates of a standard vector sequence corresponding to $\rho$ and to a fixed (countable) orthonormal set $\mathcal{E}$.

The problem that occurs when $\rho<0$ is that only finitely many of the standard vectors defined in this manner have norm equal to 1 , which is one of the defining conditions for a set with the CIP $-\rho$ property.

More specifically: for $i \geq 1$ one can define the weights

$$
r_{i}=\frac{\rho}{1+(i-1) \rho}, s_{i}=\sqrt{1-r_{i}^{2}} .
$$

Having defined the vectors $x_{k}=x_{k}[\rho]$ as in Section 2.5, we see that there is a maximal value of $N$ for which $\left\{x_{1}, x_{2}, \ldots, x_{N}\right\}$ all have norm equal to one, while $\left\|x_{N+1}\right\|>1$. Such a unique $N$ is said to be admitted by $\rho$ or $\rho$-admissible and we write $N=\operatorname{ad}(\rho)$. We observe that $2 \leq N=\operatorname{ad}(\rho)$ if and only if $\frac{-1}{N-1} \leq \rho<\frac{-1}{N}$, and that this happens if and only if $N=\left\lfloor\frac{-1}{\rho}\right\rfloor+1$. In other words, $N=\operatorname{ad}(\rho)=\left\lfloor\frac{-1}{\rho}\right\rfloor+1$. Also note that if $N=\operatorname{ad}(\rho)$, then $\operatorname{ad}\left(r_{i}\right)=\left\lfloor\frac{-1}{\rho}\right\rfloor+(2-i)$ where $1 \leq i \leq N-1$, and hence $\operatorname{ad}\left(r_{i+1}\right)=\operatorname{ad}\left(r_{i}\right)-1$ for all 
$1 \leq i \leq N-2$. As $r_{i}=\frac{\rho}{1+(i-1) \rho}$, it is clear that the sequence $\left(r_{i}\right)_{i=1}^{\operatorname{ad}(\rho)-1}$ is decreasing on its domain, i.e., $r_{i+1}<r_{i}$ whenever $i+1 \leq \operatorname{ad}(\rho)-1$. We leave it to the interested reader to check that if $\operatorname{ad}(\rho)=N$, then

$$
\frac{-1}{N-1} \leq r_{1}<\frac{-1}{N}, \frac{-1}{N-2} \leq r_{2}<\frac{-1}{N-1}, \ldots,-1 \leq r_{N-1}<\frac{-1}{2} .
$$

and in particular $r_{N}:=\frac{r_{N-1}}{1+r_{N-1}}=\frac{\rho}{1+(N-1) \rho}<-1$ if $\rho \neq \frac{-1}{N-1}$ or equivalently, if $r_{N-1} \neq-1$. But $\left|r_{N}\right|>1$ implies that $\left\|x_{N}\right\|>1$, which precludes $x_{N}$ from belonging to a set with the CIP $-\rho$ property.

2.14. Lemma. Let $\mathcal{H}$ be a Hilbert space and $\mathcal{E}_{N}=\left(e_{n}\right)_{n=1}^{N}$ a finite orthonormal sequence of vectors in $\mathcal{H}$, where $N \leq \operatorname{ad}(\rho)$ if $-1<\rho<0$ but $\rho \neq \frac{-1}{N-1}$ and $N<\operatorname{ad}(\rho)$ if $\rho=\frac{-1}{N-1}$. If $\Gamma\left(\mathcal{E}_{N}, r\right)=\left(x_{n}\right)_{n=1}^{N}$, then $\left\langle x_{i}, x_{j}\right\rangle=\rho+\delta_{i, j}(1-\rho)$ for all $1 \leq i, j \leq N$, where $\delta_{i, j}$ denotes the Kronecker delta. Moreover, $\Gamma\left(\mathcal{E}_{N}, \rho\right)$ is linearly independent and $\operatorname{span} \Gamma\left(\mathcal{E}_{N}, \rho\right)=\operatorname{span} \mathcal{E}_{N}$.

Proof. The proof of this result is identical to that of Lemma 2.6 (a).

As before, we observe that the Gram-Schmidt process applied to the sequence $\Gamma\left(\mathcal{E}_{N}, \rho\right)=$ $\left(x_{n}\right)_{n=1}^{N}$ returns the orthonormal sequence $\mathcal{E}=\left(e_{n}\right)_{i=1}^{N}$.

The following result is the analogue of Lemma 2.8 (and the proof of that result applies equally well to this case) for the case where $-1<\rho<0$. The only difference between the two results is that we require $N<\operatorname{ad}(\rho)$ in order to ensure that the norm of the vectors we consider is one.

2.15. Lemma. Let $\mathcal{H}$ be a Hilbert space and $\mathcal{E}_{N}=\left\{e_{n}\right\}_{n=1}^{N}$ be an orthonormal subset of $\mathcal{H}$, where $N<\operatorname{ad}(\rho)$. If $\Gamma\left(\mathcal{E}_{N}, \rho\right)=\left(x_{n}\right)_{n=1}^{N}$, then $\left\langle y, x_{n}\right\rangle=\rho$ for all $1 \leq n \leq N$ if and only if $\left\langle y, e_{1}\right\rangle=r_{1}$ and $\left\langle y, e_{n}\right\rangle=s_{1} \cdots s_{n-1} r_{n}$ for all $1<n \leq N$. Moreover, if we let $y_{N}=\sum_{n=1}^{N}\left\langle y, e_{n}\right\rangle e_{n}$, then $\left\langle y_{N}, y_{N}\right\rangle=1-s_{1}^{2} \cdots s_{N}^{2}=1-\left(1-r_{1}\right)\left(1+r_{N}\right)$.

2.16. Proposition. Let $\mathcal{H}$ be a Hilbert space and $-1<\rho<0$.

(a) Let $N \in \mathbb{N}$ with $N=\operatorname{ad}(\rho)$ but $\rho \neq \frac{-1}{N-1}$, and let $\mathcal{E}_{N}=\left\{e_{i}\right\}_{i=1}^{N}$ be an orthonormal subset of $\mathcal{H}$. Then $\Gamma\left(\mathcal{E}_{N}, \rho\right)=\left(x_{i}\right)_{i=1}^{N}$ is a maximal family of unit vectors in $\mathcal{H}$ for which any two distinct vectors have constant inner product $\rho$.

(b) Let $\rho=\frac{-1}{N-1}$, where $N \in \mathbb{N}$ so that $\operatorname{ad}(\rho)=N, \mathcal{E}_{N}=\left\{e_{i}\right\}_{i=1}^{N}$ be an orthonormal subset of $\mathcal{H}$, and $\Gamma\left(\mathcal{E}_{N}, \rho\right)=\left(x_{i}\right)_{i=1}^{N}$. Then $x_{N}=-\left(x_{1}+\cdots+x_{N-1}\right)$ and $\Gamma\left(\mathcal{E}_{N}, \rho\right)$ is a maximal family of unit vectors in $\mathcal{H}$ for which any two distinct vectors have constant inner product $\rho$.

\section{Proof.}

(a) Suppose by contradiction that $y \in \mathcal{H}$ is a unit vector and $\left\langle y, x_{i}\right\rangle=\rho$ for all $1 \leq i \leq$ $N$. Note that $N-1<N=\operatorname{ad}(\rho)$. It thus follows from Lemma 2.15 that $\left\langle y, e_{1}\right\rangle=r_{1}$ and $\left\langle y, e_{i}\right\rangle=s_{1} \cdots s_{i-1} r_{i}$ for all $1<i \leq N-1$. Since $-1<r_{N-1}<\frac{-1}{2}$ (because $\left.\rho \neq \frac{-1}{N-1}\right)$, we can actually define $r_{N}:=\frac{r_{N-1}}{1+r_{N-1}}$ and see that $r_{N}<-1$. It now follows from the proof of Lemma 2.15 that $\left\langle y, e_{N}\right\rangle=s_{1} \cdots s_{N-1} r_{N}$. Thus we can 
write $y=\sum_{i=1}^{N}\left\langle y, e_{i}\right\rangle e_{i}+y_{\perp}$, where $y_{\perp} \in \mathcal{H} \ominus \operatorname{span} \mathcal{E}$. This leads to a contradiction, for we will then obtain

$$
\begin{aligned}
1 & =\langle y, y\rangle \\
& =\left(r_{1}^{2}+s_{1}^{2} r_{2}^{2}+\cdots+s_{1}^{2} \cdots s_{N-2}^{2} r_{N-1}^{2}+s_{1}^{2}+\cdots s_{N-1}^{2} r_{N}^{2}\right)+\left\|y_{\perp}\right\|^{2}, \\
& =\left(r_{1}^{2}+s_{1}^{2} r_{2}^{2}+\cdots+s_{1}^{2} \cdots s_{N-2}^{2}\left(1-s_{N-1}^{2}\right)+s_{1}^{2}+\cdots s_{N-1}^{2} r_{N}^{2}\right)+\left\|y_{\perp}\right\|^{2}, \\
& =\left(\left(r_{1}^{2}+s_{1}^{2} r_{2}^{2}+\cdots+s_{1}^{2} \cdots s_{N-2}^{2}\right)-s_{1}^{2} \cdots s_{N-1}^{2}+s_{1}^{2} \cdots s_{N-1}^{2} r_{N}^{2}\right)+\left\|y_{\perp}\right\|^{2}, \\
& =\left(1-s_{1}^{2} \cdots s_{N-1}^{2}\left(1-r_{N}^{2}\right)\right)+\left\|y_{\perp}\right\|^{2}, \\
& =\left(1-s_{1}^{2} \cdots s_{N-1}^{2}\left(1-r_{N}^{2}\right)\right)+\left\|y_{\perp}\right\|^{2}, \\
& =\left(1-\left(1-r_{1}\right)\left(1+r_{N}\right)\right)+\left\|y_{\perp}\right\|^{2},
\end{aligned}
$$

implying that

$$
\left\|y_{\perp}\right\|^{2}=\left(1-r_{1}\right)\left(1+r_{N}\right),
$$

which yields $\left\|y_{\perp}\right\|<0$ because $1+r_{N}<0$, which is impossible. This contradiction proves the assertion.

(b) Suppose that $y \in \mathcal{H}$ is a unit vector and $\left\langle y, x_{i}\right\rangle=\rho$ for all $1 \leq i \leq N-1$. We need to show that $y=x_{N}=-\left(x_{1}+\cdots+x_{N-1}\right)$. To this end, as $N-1<N=\operatorname{ad}(\rho)$, we see from Lemma 2.6 (a) that $\left\langle y, e_{1}\right\rangle=r_{1}$ and $\left\langle y, e_{i}\right\rangle=s_{1} \cdots s_{i-1} r_{i}$ for all $1<i \leq N-1$. Note that $r_{N-1}=-1$, and hence $s_{N-1}=0$, because $\rho=\frac{-1}{N-1}$. Thus we can write $y=\sum_{i=1}^{N-1}\left\langle y, e_{i}\right\rangle e_{i}+y_{\perp}$, where $y_{\perp} \in \mathcal{H} \ominus \operatorname{span}\left\{e_{i}\right\}_{i=1}^{N-1}$. Thus

$1=\langle y, y\rangle$

$$
\begin{aligned}
& =\left(r_{1}^{2}+s_{1}^{2} r_{2}^{2}+\cdots+s_{1}^{2} \cdots s_{N-3}^{2} r_{N-2}^{2}+s_{1}^{2}+\cdots s_{N-2}^{2} r_{N-1}^{2}\right)+\left\|y_{\perp}\right\|^{2} \\
& =\left(r_{1}^{2}+s_{1}^{2} r_{2}^{2}+\cdots+s_{1}^{2} \cdots s_{N-3}^{2}\left(1-s_{N-2}^{2}\right)+s_{1}^{2}+\cdots s_{N-2}^{2} r_{N-1}^{2}\right)+\left\|y_{\perp}\right\|^{2}, \\
& =\left(\left(r_{1}^{2}+s_{1}^{2} r_{2}^{2}+\cdots+s_{1}^{2} \cdots s_{N-3}^{2}\right)-s_{1}^{2} \cdots s_{N-2}^{2}+s_{1}^{2} \cdots s_{N-2}^{2} r_{N-1}^{2}\right)+\left\|y_{\perp}\right\|^{2}, \\
& =\left(1-s_{1}^{2} \cdots s_{N-2}^{2}\left(1-r_{N-1}^{2}\right)\right)+\left\|y_{\perp}\right\|^{2}, \\
& =(1-0)+\left\|y_{\perp}\right\|^{2},
\end{aligned}
$$

implying that

$$
\left\|y_{\perp}\right\|^{2}=0
$$

which in turn yields $y_{\perp}=0$. Therefore, $y=\sum_{i=1}^{N-1}\left\langle y, e_{i}\right\rangle e_{i}$. This together with $\left\langle y, e_{1}\right\rangle=r_{1}$ and $\left\langle y, e_{i}\right\rangle=s_{1} \cdots s_{i-1} r_{i}$ for all $1<i \leq N-1$ and the fact that

$x_{N}=r_{1} e_{1}+s_{1} r_{2} e_{2}+s_{1} s_{2} r_{3} e_{3}+\cdots+s_{1} s_{2} s_{3} \cdots s_{N-2} r_{N-1} e_{N-1}+s_{1} s_{2} s_{3} \cdots s_{N-1} e_{N}$

shows that $y=x_{N}$. Clearly, $x_{N}=c_{1} x_{1}+\cdots+c_{N-1} x_{N-1}$ for some $c_{i} \in \mathbb{C}(1 \leq i \leq$ $N-1)$. Then for each $1 \leq i \leq N$, we have

$$
\left\langle x_{N}, x_{i}\right\rangle=\left\langle c_{1} x_{1}+c_{2} x_{2}+\cdots+c_{N-1} x_{N-1}, x_{i}\right\rangle .
$$


This clearly gives rise to

$$
\begin{aligned}
& 1=c_{1} r+c_{2} r+\cdots+c_{N-1} \rho, \\
& r=c_{i}+\sum_{i \neq k=1}^{N-1} c_{k} \rho,(1 \leq i \leq N-1)
\end{aligned}
$$

from which we easily see that $c_{i}=-1$ for all $1 \leq i \leq N-1$. In other words, $y=x_{N}=-\left(x_{1}+\cdots+x_{N-1}\right)$, as desired. This proves the assertion.

We are now in a position to establish the converse to Proposition 2.16.

2.17. Theorem. Let $-1<\rho<0$ and suppose that $\mathcal{H}$ is a Hilbert space. Suppose also that $\Theta \subseteq \mathcal{H}$ is a set of unit vectors such that $x \neq y \in \Theta$ implies that $\langle x, y\rangle=\rho$.

(a) The set $\Theta$ is finite and $|\Theta| \leq \operatorname{ad}(\rho)$.

(b) The set $\Theta$ is linearly dependent if and only if $\rho=\frac{-1}{|\Theta|-1}$, in which case $\sum_{x \in \Theta} x=0$, $\operatorname{dim} \operatorname{span} \Theta=|\Theta|-1$, and for any $x \in \Theta, \Theta \backslash\{x\}=\Gamma\left(\mathcal{E}_{x}, \rho\right)$, where $\mathcal{E}_{x}$ is an orthonormal basis for span $\Theta$ obtained by applying the Gram-Schmidt process to $\Theta \backslash\{x\}$.

(c) If $\Theta$ is linearly independent, equivalently, $\rho \neq \frac{-1}{|\Theta|-1}$, then $\Theta=\Gamma(\mathcal{E}, \rho)$, where $\mathcal{E}=$ $\left\{e_{n}\right\}_{n=1}^{|\Theta|}$ is an orthonormal basis for span $\Theta$ obtained by applying the Gram-Schmidt process to $\Theta$.

(d) The set $\Theta$ is maximal if and only if either $|\Theta|=\operatorname{ad}(\rho)$ or $\operatorname{dim} \mathcal{H}=|\Theta|<\operatorname{ad}(\rho)$.

\section{Proof.}

(a) We argue by contradiction. Suppose, to the contrary, that $|\Theta|>\operatorname{ad}(\rho)$ and choose a subset $\left\{x_{i}\right\}_{i=1}^{M}$ of $\Theta$, where $M=\operatorname{ad}(\rho)+1$. Clearly, $1+(M-1) \rho \neq 0$, for otherwise $\operatorname{ad}(\rho)=M$, which is impossible. This implies that

$$
\operatorname{det} R_{M}=(1-\rho)^{M-1}(1+(M-1) \rho) \neq 0,
$$

where $R_{M} \in \mathbb{M}_{M}(\mathbb{R})$ is as in the proof of Theorem 2.12. Consequently, we see that $\left\{x_{i}\right\}_{i=1}^{M}$ is linearly independent. Apply the Gram-Schmidt process to $\left\{x_{i}\right\}_{i=1}^{M}$ to obtain the orthonormal subset $\mathcal{E}=\left\{e_{i}\right\}_{i=1}^{M}$ of $\mathcal{H}$. Again, just as we saw in the proof of part (a) of Theorem 2.12, we get that $\left\{x_{i}\right\}_{i=1}^{M}=\Gamma(\mathcal{E}, \rho)$. From this it follows that $M=\operatorname{ad}(\rho)+1 \leq \operatorname{ad}(\rho)$, which is a contradiction. Thus $|\Theta| \leq \operatorname{ad}(\rho)$, as desired.

(b) Suppose that $\Theta$ is linearly dependent. Let $N:=|\Theta| \leq \operatorname{ad}(\rho)$ and $\Theta=\left\{x_{i}\right\}_{i=1}^{N}$. Once again, with $R_{N}$ as in the proof of Theorem 2.12, we see that det $R_{N}=(1-\rho)^{N-1}(1+$ $(N-1) \rho)=0$, which yields $\rho=\frac{-1}{N-1}=\frac{-1}{|\Theta|-1}$, proving the "only if" implication. As for the "if" implication, if $\rho=\frac{-1}{|\Theta|-1}$, setting $N:=|\Theta|$ with $R_{N}$ as in the above, we see that $\operatorname{det} R_{N}=0$, which in turn, in view of the proof of Theorem 2.12, implies that $\Theta=\left\{x_{i}\right\}_{i=1}^{N}$ is linearly dependent, which is what we want. Now since $\Theta=$ $\left\{x_{i}\right\}_{i=1}^{N}$ is linearly dependent, if necessary by renaming $x_{i}{ }^{\prime} \mathrm{s}(1 \leq i \leq N)$, we may choose the scalars $c_{1}, c_{2}, \ldots, c_{N-1} \in \mathbb{C}$ such that $x_{N}=c_{1} x_{1}+c_{2} x_{2}+\cdots+c_{N-1} x_{N-1}$. But, just as we saw in the proof of Proposition 2.16, this yields $c_{i}=-1$ for all $1 \leq i \leq N-1$. That is, $\sum_{i=1}^{N} x_{i}=\sum_{x \in \Theta} x=0$. Finally, the set $\left\{x_{i}\right\}_{i=1}^{N-1}$ is linearly independent because $\operatorname{det} R_{N-1} \neq 0$. Therefore, dim span $\Theta=|\Theta|-1$. Choosing any $x \in \Theta$, it is plain that $\Theta \backslash\{x\}$ is a basis for span $\Theta$. Thus we have $\Theta \backslash\{x\}=$ $\Gamma\left(\mathcal{E}_{x}, \rho\right)$, where $\mathcal{E}_{x}$ is an orthonormal basis for span $\Theta$ obtained by applying the Gram-Schmidt process to $\Theta \backslash\{x\}$. This proves the assertion. 
(c) If $\Theta$ is linearly independent, apply the Gram-Schmidt process to $\left\{x_{i}\right\}_{i=1}^{N}$ to obtain an orthonormal basis $\mathcal{E}=\left\{e_{i}\right\}_{i=1}^{N}$ for span $\Theta$. Once again just as we saw in the proof of part (a) of Theorem 2.12, we conclude that $\left\{x_{i}\right\}_{i=1}^{N}=\Gamma(\mathcal{E}, \rho)$, as desired.

(d) Suppose first that $\Theta$ is maximal but $|\Theta| \neq \operatorname{ad}(\rho)$ so that $|\Theta|<\operatorname{ad}(\rho)$. We need to show that $\operatorname{dim} \mathcal{H}=|\Theta|$. Note that $\rho \neq \frac{-1}{|\Theta|-1}$ because $|\Theta| \neq \operatorname{ad}(\rho)$. Thus, $\Theta$ is linearly independent, and hence $|\Theta| \leq \operatorname{dim} \mathcal{H}$. We extract a contradiction from $\operatorname{dim} \mathcal{H}>|\Theta|$. As $\Theta$ is linearly independent, by what we saw in the preceding paragraph $\Theta=\Gamma(\mathcal{E}, \rho)$, where $\mathcal{E}$ is an orthonormal basis for span $\Theta$ obtained by applying the Gram-Schmidt process to $\Theta$. But $\operatorname{dim} \mathcal{H}>\operatorname{dim} \operatorname{span} \Theta=|\Theta|=|\mathcal{E}|$ and $|\Theta|<\operatorname{ad}(\rho)$. So we may choose a unit vector $e \in \mathcal{H}$ such that $\mathcal{E}_{0}=\mathcal{E} \cup\{e\}$ is orthonormal. Since $|\Theta|<\operatorname{ad}(\rho)$, we see that $\Theta=\Gamma(\mathcal{E}, \rho) \subsetneq \Gamma\left(\mathcal{E}_{0}, \rho\right)$, contradicting the maximality of $\Theta$. This proves the "only if" implication. To prove the "if" implication assume first that $|\Theta|=\operatorname{ad}(\rho)$. If $\rho \neq \frac{-1}{|\Theta|-1}$, then $\Theta$ is linearly independent and again as we saw in the preceding paragraph, $\Theta=\Gamma(\mathcal{E}, \rho)$, where $\mathcal{E}$ is an orthonormal basis for span $\Theta$ obtained by applying the Gram-Schmidt process to $\Theta$. It now follows from Proposition 2.16 that $\Theta$ is maximal. If $\rho=\frac{-1}{|\Theta|-1}$, then, by what we just showed in the above, $\Theta$ is linearly dependent but $\Theta \backslash\{x\}$ is linearly independent for any $x \in \Theta$. Fix $x \in \Theta$ and write $\Theta \backslash\{x\}=\Gamma(\mathcal{E}, \rho)$, where $\mathcal{E}$ is an orthonormal basis for span $\Theta$ obtained by applying the Gram-Schmidt process to $\Theta \backslash\{x\}$. It now follows from the proof of Proposition 2.16 that $\Theta$ is maximal. Lastly, if $\operatorname{dim} \mathcal{H}=|\Theta|<\operatorname{ad}(\rho)$, again $\Theta$ is linearly independent, and hence $\Theta=\Gamma(\mathcal{E}, \rho)$, where $\mathcal{E}$ is an orthonormal basis for span $\Theta=\mathcal{H}$ obtained by applying the GramSchmidt process to $\Theta$. Now the maximality of $\Theta$ follows from Proposition 2.16(a). This completes the proof.

\section{IRREDUCIBLE, SELFADJOINT SEMIGROUPS}

3.1. We next turn our attention to a somewhat different problem. Our present goal is to characterize those two-element sets $\Omega \subseteq \mathbb{C}$ which can appear as the admissible set of a selfadjoint, irreducible semigroup $\mathcal{S}$ of operators. Our main result is Theorem 3.9 below, which shows that when the underlying Hilbert space is infinite-dimensional and separable, $\Omega$ must be of the form $\Omega=\left\{0, \frac{1}{p}\right\}$ for some positive integer $p$. We shall arrive at that result through a series of intermediate results.

The following result is stated explicitly as Corollary 2.1.6 in [4] for semigroups acting on finite-dimensional Hilbert spaces.

3.2. Lemma. Let $\mathcal{H}$ be a complex Hilbert space of dimension at least two, $\mathcal{S} \subseteq \mathcal{B}(\mathcal{H})$ be an irreducible semigroup, and $x, y \in \mathcal{H}$ be fixed, non-zero vectors. Suppose that $\varphi: \mathcal{B}(\mathcal{H}) \rightarrow \mathbb{C}$ is the vector functional defined by $\varphi(T)=\langle T x, y\rangle$ for all $T \in \mathcal{B}(\mathcal{H})$.

Then $\varphi(S):=\{\varphi(S): S \in \mathcal{S}\}$ has at least two elements.

Proof. Suppose that $\varphi(\mathcal{S})=\{\alpha\}$. The irreducibility of $\mathcal{S}$ implies that of $\mathcal{S}^{*}$, since $\mathcal{M} \in$ Lat $\mathcal{S}^{*}$ implies that $\mathcal{M}^{\perp} \in$ Lat $\mathcal{S}$. Thus, we can find $T_{1}, T_{2} \in \mathcal{S}$ such that

$$
T_{1}^{*} y \neq T_{2}^{*} y \text {. }
$$

Consider next $\alpha=\varphi\left(T_{1} S\right)=\varphi\left(T_{2} S\right)$ for all $S \in \mathcal{S}$, so that

$$
\left\langle S x,\left(T_{1}-T_{2}\right)^{*} y\right\rangle=0 \quad \text { for all } S \in \mathcal{S} .
$$


But then $\overline{\mathcal{S} x}$ is perpendicular to $\left(T_{1}-T_{2}\right)^{*} y \neq 0$, contradicting the irreducibility of $\mathcal{S}$.

3.3. Proposition. Let $\mathcal{H}$ be a complex Hilbert space and $\mathcal{S} \subseteq \mathcal{B}(\mathcal{H})$ be a selfadjoint, irreducible semigroup of operators on $\mathcal{H}$. Suppose furthermore that $\Omega$ is a bounded, admissible set for $\mathcal{S}$. Then $\mathcal{S}$ is bounded; in fact, $S \in \mathcal{S}$ implies that $\|S\| \leq 1$.

It follows that if $\mathcal{S}$ is a group with these properties, then $\mathcal{S}$ consists of unitary operators.

Proof. In the case where $\operatorname{dim} \mathcal{H}=1$, this is straightforward. Suppose therefore that $\operatorname{dim} \mathcal{H} \geq 2$, and let $\xi \in \mathcal{H}$ denote a norm-one admissible vector corresponding to the bounded admissible set $\Omega$.

Suppose that $0 \leq T \in \mathcal{S}$ with $\|T\|>1$. Denoting by $E_{T}(\cdot)$ the spectral projection function for $T$, we see that there must exist $\delta>0$ so that $Q:=E_{T}([1+\delta,\|T\|]) \neq 0$. By the functional calculus for normal operators, we also have that for all $k \geq 1$,

$$
T^{k} \geq Q T^{k} \geq(1+\delta)^{k} Q \text {. }
$$

The irreducibility of $\mathcal{S}$, combined with the fact that $Q \neq 0$ implies that there exists $S \in \mathcal{S}$ such that $Q S \xi \neq 0$.

Observe next that $0 \leq S^{*} T^{k} S \in \mathcal{S}$ and so $0 \leq\left\langle S^{*} T^{k} S \xi, \xi\right\rangle \in \Omega$ for all $k \geq 1$. But then for each $k \geq 1$,

$$
\begin{aligned}
\left\langle S^{*} T^{k} S \xi, \xi\right\rangle & =\left\langle T^{k} S \xi, S \xi\right\rangle \\
& \geq\left\langle\left(Q T^{k}\right) S \xi, S \xi\right\rangle \\
& \geq\left\langle\left((1+\delta)^{k} Q\right) S \xi, S \xi\right\rangle \\
& =(1+\delta)^{k}\langle Q S \xi, Q S \xi\rangle \\
& =(1+\delta)^{k}\|Q S \xi\|^{2} .
\end{aligned}
$$

Since $\lim _{k \rightarrow \infty}(1+\delta)^{k}\|Q S \xi\|^{2}=\infty$, this contradicts the boundedness of $\Omega$.

Thus, $0 \leq T \in \mathcal{S}$ implies that $\|T\| \leq 1$. But if $R \in \mathcal{S}$ and $\|R\|>1$, then $0<R^{*} R \in \mathcal{S}$ and $\left\|R^{*} R\right\|=\|R\|^{2}>1$, a contradiction. This completes the proof.

We observe that the same result fails if we simply drop the condition that $\mathcal{S}$ be selfadjoint.

3.4. Example. For each $N \geq 1$, let $B_{1}\left(\mathbb{C}^{N}\right)=\left\{T \in \mathbb{M}_{N}(\mathbb{C}):\|T\| \leq 1\right\}$. Let us identify $\mathcal{H}$ with $\ell^{2}(\mathbb{N})$ and consider

$$
B_{1}(\mathcal{H}):=\cup_{N=1}^{\infty}\left\{B \oplus 0^{(\infty)}: B \in B_{1}\left(\mathbb{C}^{N}\right)\right\} \subseteq \mathcal{B}(\mathcal{H}) .
$$

Define $K=\operatorname{diag}\left(\frac{1}{n}\right)_{n=1}^{\infty}$ and $L=\operatorname{diag}(n)_{n=1}^{\infty}$. It is understood that $L \notin \mathcal{B}(\mathcal{H})$. For $N \geq 1$, let $K_{N}=\operatorname{diag}_{n=1}^{N}\left(\frac{1}{n}\right) \in \mathbb{M}_{N}(\mathbb{C})$.

Let

$$
\begin{aligned}
\mathcal{S} & =\left\{K T L: T \in \mathcal{B}_{1}(\mathcal{H})\right\} \\
& =\cup_{N=1}^{\infty}\left\{K_{N} B K_{N}^{-1} \oplus 0^{(\infty)}: B \in \mathcal{B}_{1}\left(\mathbb{C}^{N}\right)\right\} .
\end{aligned}
$$

It is readily verified that $\mathcal{S}$ is an irreducible semigroup in $\mathcal{B}(\mathcal{H})$ (in $\operatorname{fact}, \operatorname{span}(\mathcal{S})$ is weak-operator topology dense in $\mathcal{B}(\mathcal{H})$, by virtue of containing each matrix unit $\left.E_{i, j}\right)$.

Moreover, 
(a) For each $m \geq 1,\left\{\left\langle S e_{m}, e_{m}\right\rangle: S \in \mathcal{S}\right\}=\mathbb{D}:=\{z \in \mathbb{C}:|z| \leq 1\}$. In particular, the admissible set corresponding to $e_{m}$ is bounded.

(b) For each $1 \leq i, j \leq N$ and for each $S \in \mathcal{S}$,

$$
\begin{aligned}
\mid\left\langle S e_{i}, e_{j}\right\rangle & =\left|\left\langle K_{N} B K_{N}^{-1} e_{i}, e_{j}\right\rangle\right| \quad \text { for some } B \in B_{1}\left(\mathbb{C}^{N}\right) \\
& =\mid\left\langle B\left(K_{N}^{-1} e_{i},\left(K_{N}^{*} e_{j}\right)\right\rangle\right| \\
& \leq\|B\|\left\|K_{N}^{-1} e_{i}\right\|\left\|K_{N}^{*} e_{j}\right\| \\
& \leq N .
\end{aligned}
$$

(c) For each $N \geq 1, N E_{1, N} \in \mathcal{S}$, and so $\mathcal{S}$ is not bounded, though for each $1 \leq i, j$, the set $\left\{\left\langle S e_{i}, e_{j}\right\rangle: S \in \mathcal{S}\right\}$ is bounded, with the bound depending upon $\max (i, j)$.

We can even do a bit better. Let $\mathcal{E}=\left\{E_{i, j}: 1 \leq i, j\right\} \cup\{0\}$, so that $\mathcal{E}$ is an irreducible semigroup in $\mathcal{B}(\mathcal{H})$. Again, let $\mathcal{F}:=\{K E L: E \in \mathcal{E}\}$. Then $\mathcal{F}$ is an irreducible semigroup in $\mathcal{B}(\mathcal{H}), \mathcal{F}$ is unbounded $\left(n E_{1, n} \in \mathcal{F}\right.$ for all $\left.n \geq 1\right)$, and for any $m \geq 1$, the basis vector $e_{m}$ is an admissible vector corresponding to the admissible set $\{0,1\}$ for $\mathcal{F}$. In other words, $\mathcal{F}$ is an unbounded, irreducible semigroup which admits a two-element admissible set $\Omega=\{0,1\}$.

3.5. Example 3.4 may suggest that an irreducible semigroup $\mathcal{S}$ on which a linear functional has bounded values may at least be unboundedly similar to a bounded semigroup; i.e. that there exists an injective linear transformation $T$ with an appropriate dense domain $\mathcal{D}$ such that $T \mathcal{D}$ is also dense in $\mathcal{H}$, and the semigroup $T \mathcal{S} T^{-1}$ (defined on $T \mathcal{D}$ ) is bounded.

The following example shows that such an unbounded similarity need not exist even in the case where $\mathcal{S}$ consists of rank-one operators and admits many states $\tau$ for which the corresponding admissible sets $\tau(\mathcal{S})$ have cardinality two.

3.6. Example. Let $\left\{e_{i}\right\}$ be an orthonormal basis for $\mathcal{H}$. Let $\left\{E_{i, j}\right\}$ be the set of basic operators and define $\mathcal{S}$ to be the semigroup consisting of 0 , and rank-one operators of the form

$$
\sum_{i \in F} E_{i, j}, j \in \mathbb{N}
$$

where $F$ is a finite set.

Note that for any fixed $i, j \geq 1,\left\langle S e_{i}, e_{j}\right\rangle \in\{0,1\}$, so that $\mathcal{S}$ has many 2-element admissible sets, and $\mathcal{S}$ is, of course, irreducible. We claim that no injective linear transformation $T$ exists such that the semigroup $\varphi(\mathcal{S})$ defined by

$$
\varphi(S) T=T S \quad \text { for } S \in \mathcal{S}
$$

is bounded.

The domain $\mathcal{D}$ of $T$ contains the (non-closed) linear span $\mathcal{M}$ of the ranges of the $E_{i, j}$, i.e. $\mathcal{M}=\operatorname{span}\left\{e_{i}: i \in \mathbb{N}\right\}$, so that we can assume with no loss of generality that $\mathcal{D}=\mathcal{M}$. Let $f_{i}=T e_{i}$ and let $\varphi\left(E_{i, j}\right)=F_{i, j}$. It follows from the equations

$$
E_{i, j} e_{k}=\delta_{j, k} e_{i}
$$

for all $i, j, k \geq 1$ that

Now $F_{i, j} f_{j}=f_{i}$ yields

$$
F_{i, j} f_{k}=\delta_{j, k} f_{i} .
$$

$$
\left\|F_{i, j}\right\| \geq \frac{\left\|f_{i}\right\|}{\left\|f_{j}\right\|} .
$$


If $\sup _{k}\left\|f_{k}\right\|=\infty$, or if $\inf _{k}\left\|f_{k}\right\|=0$, this inequality implies the unboundedness of $\varphi(\mathcal{S})$, and we are done. So to prove the claim we will assume the existence of strictly positive scalars $m$ and $M$ such that for all $k \geq 1$,

$$
m \leq\left\|f_{k}\right\| \leq M
$$

Thus $\left\{f_{k}\right\}_{k}$ has a weakly convergent subsequence $\left\{f_{k_{n}}\right\}_{n}$ with weak limit $g$. We next distinguish two cases:

- CASE ONE. Suppose that $g \neq 0$. Then

$$
\lim _{n}\left\langle f_{k_{n}}, g\right\rangle=\|g\|^{2}
$$

Choose a subsequence of $\left\{f_{k_{n}}\right\}_{n}$ (which we still denote by $\left.\left\{f_{k_{n}}\right\}_{n}\right)$, with

$$
\left|\left\langle f_{k_{n}}, g\right\rangle-\|g\|^{2}\right|<\frac{1}{2^{n}}
$$

for all $n \geq 1$.

This implies that

$$
\left|\sum_{n=1}^{N}\left\langle f_{k_{n}}, g\right\rangle\right| \geq N\left\|g^{2}\right\|-1
$$

for every integer $N$. Now $\sum_{n=1}^{N} f_{k_{n}, 1} \in \varphi(\mathcal{S})$ and

$$
\begin{aligned}
\left\|\sum_{n=1}^{N} F_{k_{n}, 1}\right\| & \geq \frac{\left|\left\langle\sum_{n=1}^{N} F_{k_{n}, 1} f_{1}, g\right\rangle\right|}{\left\|f_{1}\right\|\|g\|} \\
& \geq \frac{\left|\left\langle\sum_{n=1}^{N} f_{k_{n}}, g\right\rangle\right|}{M\|g\|} \\
& \geq \frac{N\|g\|^{2}-1}{M\|g\|} .
\end{aligned}
$$

Since $N$ can be chosen arbitrarily large, we see that $\varphi(\mathcal{S})$ is unbounded.

- CASE TwO. Suppose that $g=0$.

In this case we pick a subsequence of $f_{k_{n}}$ inductively as follows.

Let $h_{1}=f_{k_{1}}$. Having chosen $h_{1}, h_{2}, \ldots, h_{j}$ from $\left\{f_{k_{n}}\right\}_{n}$, we may find $h_{j+1}$ in $\left\{f_{k_{n}}\right\}_{n}$ such that

$$
\left|\left\langle h_{j+1}, h_{i}\right\rangle\right|<\frac{1}{j \cdot 2^{j}}
$$

for $i=1,2, \ldots, j$. (This is possible since $\lim _{n}\left\langle f_{k_{n}}, h_{i}\right\rangle=0$ for each $i$.)

By construction, there is a member of $\varphi(\mathcal{S})$ with

$$
\varphi(S) f_{1}=\sum_{j=1}^{N} h_{j},
$$

so that

$$
\|\varphi(S)\| \geq \frac{\left\|\sum_{j=1}^{N} h_{j}\right\|}{M}
$$

for every integer $N$. 
But

$$
\begin{aligned}
\left\|\sum_{j=1}^{N} h_{j}\right\|^{2} & =\sum_{j=1}^{N} \sum_{i=1}^{N}\left\langle h_{i}, h_{j}\right\rangle \\
& =\left|\sum_{i=1}^{N}\left\|h_{i}\right\|^{2}+\sum_{1 \leq i \neq j \leq N}\left\langle h_{i}, h_{j}\right\rangle\right| \\
& \geq M N-2 \sum_{j=2}^{N} \sum_{i=1}^{j-1}\left|\left\langle h_{i}, h_{j}\right\rangle\right| \\
& \geq M N-2 \sum_{j=2}^{N} \sum_{i=1}^{j-1} \frac{1}{j \cdot 2^{j}} \\
& =M N-2 .
\end{aligned}
$$

Once again, the fact that $N$ may be chosen arbitrarily large implies that $\varphi(\mathcal{S})$ is unbounded.

3.7. Proposition. Let $\mathcal{H}$ be an infinite-dimensional, complex Hilbert space, $\mathcal{S} \subseteq \mathcal{B}(\mathcal{H})$ be an irreducible, selfadjoint semigroup of operators, and suppose that $\Omega \subseteq \mathbb{C}$ is a two-element admissible set for $\mathcal{S}$. Then $\Omega \subseteq[0,1]$.

Proof. Let $\xi$ be a norm-one admissible vector corresponding to $\Omega$. First observe that by Proposition 3.3 above, $\mathcal{S}$ is bounded in norm by 1 ; that is, if $S \in \mathcal{S}$, then $\|S\| \leq 1$. As such,

$$
|\langle S \xi, \xi\rangle| \leq\|S\|\|\xi\|^{2} \leq 1
$$

for all $S \in \mathcal{S}$; i.e. $\Omega \subseteq \overline{\mathbb{D}}=\{z \in \mathbb{C}:|z| \leq 1\}$.

Next, if $\omega \in \Omega$, then $\omega=\left\langle S_{0} \xi, \xi\right\rangle$ for some $S_{0} \in \mathcal{S}$ (by the irreducibility of $\mathcal{S}$ ), which implies that $\bar{\omega}=\left\langle S_{0}^{*} \xi, \xi\right\rangle \in \Omega$.

Moreover, given $S \in \mathcal{S}$, we have that $S^{*} \in \mathcal{S}$ and so

$$
\|S \xi\|^{2}=\left\langle S^{*} S \xi, \xi\right\rangle \in \Omega \text {. }
$$

Note that we can not have $\|S \xi\|=0$ for all $S \in \mathcal{S}$, for otherwise $\mathbb{C} \xi$ would be a non-trivial invariant subspace for $\mathcal{S}$.

Fix $T_{0} \in \mathcal{S}$ so that $T_{0} \xi \neq 0$, and let $0<\beta:=\left\|T_{0} \xi\right\|^{2} \in \Omega$. Let $\{\alpha\}=\Omega \backslash\{\beta\}$, so that $\Omega=\{\alpha, \beta\}$.

Since $\alpha \in \Omega$ implies that $\bar{\alpha} \in \Omega$ from above, and since $\alpha \neq \beta$ implies that $\bar{\alpha} \neq \bar{\beta}=\beta$, we see that in order to maintain the condition that $|\Omega|=2$, we must have $\alpha=\bar{\alpha}$, and thus $-1 \leq \alpha \leq 1$.

Next, suppose that $\alpha<0$. Since $0<\|S \xi\|^{2} \in \Omega$ for all $S \in \mathcal{S}$, we conclude that $\|S \xi\|^{2}=\beta$ for all $S \in \mathcal{S}$.

Set $\mathcal{C}=\left\{\frac{1}{\sqrt{\beta}} S \xi: S \in \mathcal{S}\right\}$. Then, by the irreducibility of $\mathcal{S}$ we see that $\overline{\text { span }} \mathcal{C}=\mathcal{H}$, and clearly $x \in \mathcal{C}$ implies that $\|x\|=1$. In particular, $\mathcal{C}$ is infinite.

Moreover, if $S_{1}, S_{2} \in \mathcal{S}$ and $S_{1} \xi \neq S_{2} \xi$, then

$$
\left\langle\frac{S_{1} \xi}{\sqrt{\beta}}, \frac{S_{2} \xi}{\sqrt{\beta}}\right\rangle \neq 1
$$

i.e., $\left\langle S_{1} \xi, S_{2} \xi\right\rangle \neq \beta$. 
But then $\left\langle S_{1} \xi, S_{2} \xi\right\rangle=\left\langle S_{2}^{*} S_{1} \xi, \xi\right\rangle \in \Omega$, so that $\left\langle S_{1} \xi, S_{2} \xi\right\rangle=\alpha$. That is,

$$
\left\langle\frac{S_{1} \xi}{\sqrt{\beta}}, \frac{S_{2} \xi}{\sqrt{\beta}}\right\rangle=\frac{\alpha}{\beta}<0
$$

for all $S_{1}, S_{2} \in \mathcal{S}$. By the results of Section 2, there can not exists infinitely many distinct unit vectors with the CIP $-\alpha$ property for $\alpha<0$. This contradiction shows that $\alpha \geq 0$. That is, $\Omega \subseteq[0,1]$.

3.8. Proposition. Let $\mathcal{H}$ be a complex, infinite-dimensional, separable Hilbert space, $\mathcal{S}=\mathcal{S}^{*} \subseteq$ $\mathcal{B}(\mathcal{H})$ be an irreducible semigroup, and suppose that $\Omega=\{\alpha, \beta\}$ is a two-element admissible set for $\mathcal{S}$. Then $0 \in \Omega$, so that $\Omega=\{0, \beta\}$ for some $0<\beta \leq 1$.

Proof. By Proposition 3.3 above, we know that $\mathcal{S}$ is bounded (in fact, $S \in \mathcal{S}$ implies that $\|S\| \leq 1$ ), and by Proposition 3.7, we know that $\Omega \subseteq[0,1]$. Let $\xi$ be an admissible unit vector corresponding to $\Omega$. After relabelling $\alpha$ and $\beta$ if necessary, we may assume without loss of generality that $0 \leq \alpha<\beta \leq 1$.

We argue by contradiction. To that end, suppose that $\alpha>0$.

We first prove that for all $S \in \mathcal{S},\|S \xi\|^{2}=\beta$. Indeed, suppose that there exists $S_{\alpha} \in \mathcal{S}$ with $\left\|S_{\alpha} \xi\right\|^{2}=\alpha$. Consider the continuous linear functional

$$
\begin{aligned}
\varphi: \mathcal{B}(\mathcal{H}) & \rightarrow \quad \mathbb{C} \\
T & \mapsto\left\langle T \xi, S_{\alpha} \xi\right\rangle .
\end{aligned}
$$

Clearly, $\varphi \neq 0$. Nevertheless, for any $S \in \mathcal{S}, \varphi(S)=\left\langle S_{\alpha}^{*} S \xi, \xi\right\rangle \in \Omega$, and thus

$$
\alpha \leq \varphi(S) \leq\|S \xi\|\left\|S_{\alpha} \xi\right\| \leq \sqrt{\beta} \sqrt{\alpha}<\beta,
$$

from which we deduce that $\varphi(S)=\alpha$. By Lemma 3.2, we conclude that $\mathcal{S}$ is reducible, which is obviously false.

Hence $S \in \mathcal{S}$ implies that $\|S \xi\|^{2}=\beta$.

Next, observe that if $S_{1}, S_{2} \in \mathcal{S}$, then $\left\langle S_{1} \xi, S_{2} \xi\right\rangle=\left\langle S_{2}^{*} S_{1} \xi, \xi\right\rangle \in \Omega$, and either $S_{1} \xi=S_{2} \xi$, or $\left\langle S_{1} \xi, S_{2} \xi\right\rangle<\left\|S_{1} \xi\right\|\left\|S_{2} \xi\right\|=\beta$, from which we conclude that $\left\langle S_{1} \xi, S_{2} \xi\right\rangle=\alpha$.

It follows that $\mathcal{C}:=\left\{\frac{S \mathcal{\zeta}}{\sqrt{\beta}}: S \in \mathcal{S}\right\}$ is a collection of unit vectors in $\mathcal{H}$ with the CIP $-\frac{\alpha}{\beta}$ property, and furthermore the irreducibility of $\mathcal{S}$ implies that this collection densely spans $\mathcal{H}$.

Note that if $\frac{S \xi}{\sqrt{\beta}} \in \mathcal{C}$ and $T \in \mathcal{S}$, then $T S \in \mathcal{S}$, and so $T \frac{S \xi}{\sqrt{\beta}}=\frac{T S \xi}{\sqrt{\beta}} \in \mathcal{C}$. Now $\mathcal{H}$ is infinite-dimensional, and $\rho:=\frac{\alpha}{\beta}>0$, so by Theorem 2.12, there exists an orthonormal basis $\mathcal{E}_{\infty}$ for $\mathcal{H}$ such that $\mathcal{C}=\Gamma\left(\mathcal{E}_{\infty}, \rho\right)=\left(x_{n}\right)_{n=1}^{\infty}$, where the $x_{n}$ 's are defined as in paragraph 2.5. Let $y_{\infty}$ be the vector defined in Lemma $2.8(\mathrm{~b})$, such that $\left\|y_{\infty}\right\|^{2}=\rho<1$, and $\left\langle y_{\infty}, x_{n}\right\rangle=\rho$ for all $n \geq 1$. As remarked at the end of the proof of that result, $y_{\infty}$ is a weak-limit of the sequence $\left(x_{n}\right)_{n=1}^{\infty}$.

Thus if $A \in \mathcal{B}(\mathcal{H})$, then $A y_{\infty}$ is the weak-limit of $\left(A x_{n}\right)_{n=1}^{\infty}$. From this we obtain that for all $S \in \mathcal{S}:$

$$
\left\langle S y_{\infty}, x_{m}\right\rangle=\lim _{n}\left\langle S x_{n}, x_{m}\right\rangle=\lim _{n}\left\langle x_{n}, S x_{m}\right\rangle=\rho,
$$

as $S x_{m} \in \mathcal{C}$ implies that $S x_{m}=x_{k}$ for some $1 \leq k$. By Proposition $2.9(\mathrm{~b})$, we may conclude that $S y_{\infty} \in \mathcal{C} \cup\left\{y_{\infty}\right\}$. On the other hand, $\left\|y_{\infty}\right\|<1$, and by Proposition 3.3, $S \in \mathcal{S}$ implies that $\|S\| \leq 1$. Hence $\left\|S y_{\infty}\right\|<1$, which forces $S y_{\infty}=y_{\infty}$. That is to say, $y_{\infty}$ is a fixed point 
of $\mathcal{S}$, and thus $\mathbb{C} y_{\infty}$ is a non-trivial invariant subspace for $\mathcal{S}$, contradicting the irreducibility of $\mathcal{S}$.

This contradiction implies that $\alpha=0$; i.e $\Omega=\{0, \beta\}$ for some $0<\beta \leq 1$.

3.9. Theorem. Let $\mathcal{H}$ be a complex, separable, infinite-dimensional Hilbert space and $\mathcal{S}$ be an irreducible, selfadjoint semigroup in $\mathcal{B}(\mathcal{H})$. Suppose that $\mathcal{S}$ admits a two-element admissible set $\Omega$. Then there exists an integer $p \geq 1$ so that $\beta=\frac{1}{p} \in \Omega$. Hence $\Omega=\left\{0, \frac{1}{p}\right\}$.

Proof. By Proposition 3.8, we know that $\Omega$ is of the form $\{0, \beta\}$ for some $0<\beta \leq 1$. Let $\xi$ be an admissible unit vector corresponding to $\Omega$. Then for any $S \in \mathcal{S}$, we know that $\|S \xi\|^{2} \in \Omega$, and that $\left\{\frac{S \xi}{\sqrt{\beta}}: S \in \mathcal{S}, S \xi \neq 0\right\}$ is an orthonormal basis for $\mathcal{H}$.

Let us denote this basis by $\mathcal{E}_{\infty}=\left(e_{n}\right)_{n=1}^{\infty}$. Let us also write $\xi=\sum_{n=1}^{\infty} \xi_{n} e_{n}$, where $\xi_{n}:=$ $\left\langle\xi, e_{n}\right\rangle$ for all $n \geq 1$.

Set $\Delta=\left\{n \in \mathbb{N}: \xi_{n} \neq 0\right\}$. Note that $S \in \mathcal{S}$ implies that $\frac{S \xi}{\sqrt{\beta}} \in \mathcal{E}_{\infty} \cup\{0\}$. Also, $\langle S \xi, \xi\rangle \in \Omega=\{0, \beta\}$. Combining these two observations with the fact for each $n \in \Delta$, there exists $R_{n} \in \mathcal{S}$ so that $e_{n}=\frac{R_{n} \xi}{\sqrt{\beta}}$, we see that

$$
\left\langle R_{n} \xi, \xi\right\rangle=\left\langle\sqrt{\beta} e_{n}, \xi\right\rangle=\sqrt{\beta} \overline{\xi_{n}} \in \Omega .
$$

But $n \in \Delta$ implies that $\xi_{n} \neq 0$, and thus $n \in \Delta$ implies that $\xi_{n}=\sqrt{\beta}$. Since $\|\xi\|^{2}=1=$ $\sum_{n \in \Delta}\left|\xi_{n}\right|^{2}=\sum_{n \in \Delta} \beta$, we see that $1=|\Delta| \beta$, or equivalently, that $\beta=\frac{1}{p}$, where $p:=|\Delta|$ is an integer.

3.10. Example. Having seen that the only possible two-element admissible sets for irreducible, selfadjoint semigroups of operators on an infinite-dimensional, separable Hilbert space are of the form $\Omega=\left\{0, \frac{1}{p}\right\}$ for some integer $p \geq 1$, let us now show that any choice of $p$ is permitted.

Let $\mathcal{H}$ be an infinite-dimensional, separable, complex Hilbert space with orthonormal basis $\left\{e_{n}\right\}_{n=1}^{\infty}$. Let $\mathcal{E}=\left\{E_{i, j}: 1 \leq i, j<\infty\right\} \cup\{0\}$, where $E_{i, j}=e_{i} \otimes e_{j}^{*}$ is the $(i, j)$-matrix unit relative to this basis. It was seen in Section 7 of [3] that $\mathcal{E}$ is an irreducible, selfadjoint semigroup of operators and that $\xi=e_{1}$ serves as an admissible vector for the admissible set $\Omega=\{0,1\}$ for $\mathcal{E}$. In particular, this shows that an example exists when $p=1$.

In fact, suppose that $p \geq 2$ is an integer and set $\xi_{p}=\frac{1}{\sqrt{p}}\left(e_{1}+e_{2}+\cdots+e_{p}\right)$. An easy calculation then shows that $\mathcal{E}$ also serves as an example of an irreducible, selfadjoint semigroup for which $\Omega_{p}:=\left\{0, \frac{1}{p}\right\}$ is an admissible set corresponding to the admissible vector $\xi_{p}$. We remark that this example is a simple extension of the example derived in Theorem 6.3 of [3]. 
3.11. Corollary. Let $\mathcal{H}$ be a complex, separable, infinite-dimensional Hilbert space and $\mathcal{U}$ be an irreducible group of unitary operators in $\mathcal{B}(\mathcal{H})$. If $\Omega$ is an admissible set for $\mathcal{U}$ with cardinality two, then $\Omega=\{0,1\}$.

Proof. The fact that $\mathcal{U}$ is a group implies that it is unital, and hence $1 \in \Omega$. The result now follows from Theorem 3.9.

In the proof of Theorem 4.1 below, we shall see that such a group is unitarily equivalent to a group of permutations.

\section{FiXED POINTS AND STRUCTURE RESUlTS}

Corollary 3.11 above tells us that if $\mathcal{U}$ is a group of unitary operators in $\mathcal{B}(\mathcal{H})$, and if $\Omega=\{\rho, 1\}$ is an admissible set for $\mathcal{U}$ with $\rho \neq 0$, then $\mathcal{U}$ admits a non-trivial invariant subspace. In fact, more is true. In this section, we demonstrate that in many cases, we can conclude the existence of fixed vectors for - and derive detailed information about the structure of - these groups simply from the value of $\rho$.

4.1. Theorem. Let $\mathcal{H}$ be a complex Hilbert space and $\mathcal{U} \subseteq \mathcal{B}(\mathcal{H})$ be a group of unitary operators on $\mathcal{H}$. Suppose furthermore that $\Omega=\{\rho, 1\}$ is an admissible set for $\mathcal{U}$, and that $\xi \in \mathcal{H}$ is $a$ corresponding admissible vector.

If $\mathcal{H}_{0}=\overline{\operatorname{span}}\{U \xi: U \in \mathcal{U}\}$, then there exists a non-zero vector $z \in \mathcal{H}_{0}$ such that $U z=z$ for all $\mathcal{U} \in \mathcal{U}$ (i.e. $z$ is a fixed point for $\mathcal{U}$ ) unless either

(a) $\mathcal{H}_{0}$ is infinite-dimensional and $\rho=0$, or

(b) $\mathcal{H}_{0}$ has finite dimension $m \geq 1$ and $\rho=-\frac{1}{m}$.

Proof. Observe that $\mathcal{H}_{0}$ is a reducing subspace for $\mathcal{U}$.

- Consider first the case where $\rho=1$, so that $\Omega=\{1\}$. It follows immediately from Lemma 3.2 that $\mathcal{U}$ is reducible.

In fact, in this case, $U \in \mathcal{U}$ implies that $\langle U \xi, \xi\rangle=1=\|\xi\|^{2}$, and since $\|U\|=1$, we must have that $U \xi=\xi$, implying that $\xi$ is a fixed point for $\mathcal{U}$, and that $\operatorname{dim} \mathcal{H}_{0}=1$.

- Next, suppose that $\rho=0$. We claim that $\left.\mathcal{U}\right|_{\mathcal{H}_{0}}$ is unitarily equivalent to a group of permutations.

Indeed, given any $U, V \in \mathcal{U}$, we have that $\langle U \xi, V \xi\rangle=\left\langle V^{*} U \xi, \xi\right\rangle \in \Omega=\{0,1\}$, so that $\mathcal{E}:=\{\mathcal{U} \xi: U \in \mathcal{U}\}$ forms an orthonormal basis for $\mathcal{H}_{0}$. Let us denote this orthonormal basis by $\mathcal{E}=\{\xi\} \cup\left\{e_{\lambda}: \lambda \in \Lambda\right\}$, where $\operatorname{dim} \mathcal{H}_{0}=1+|\Lambda|$.

For each $\alpha \in \Lambda$, fix $U_{\alpha} \in \mathcal{U}$ so that $U_{\alpha} \xi=e_{\alpha}$. Let $V \in \mathcal{U}$ be arbitrary. Then $V e_{\alpha}=V U_{\alpha} \xi \in$ $\mathcal{E}$ by the definition of $\mathcal{E}$. Since $\left.V\right|_{\mathcal{H}_{0}}$ is unitary and therefore surjective (as a map onto $\mathcal{H}_{0}$ ), we see that $V$ must permute the elements of $\mathcal{E}$; i.e., that $\left.\mathcal{U}\right|_{\mathcal{H}_{0}}$ is a group of permutation unitaries (relative to the orthonormal basis $\mathcal{E}$ ).

Suppose that $z \in \mathcal{H}_{0}$ is a fixed point for $\mathcal{U}$. Then $U_{\alpha}^{*} z=z$ for all $\alpha \in \Lambda$, and thus

$$
\langle z, \xi\rangle=\left\langle U_{\alpha}^{*} z, \xi\right\rangle=\left\langle z, U_{\alpha} \xi\right\rangle=\left\langle z, e_{\alpha}\right\rangle
$$

for all $\alpha \in \Lambda$.

If $\mathcal{H}_{0}$ has finite dimension $m \geq 1$, we then see that the vector $z=z_{0}\left(\xi+\sum_{\lambda \in \Lambda} e_{\lambda}\right)$ is a fixed point for $\mathcal{U}$, for any choice of $z_{0} \in \mathbb{C}$. (This represents the vector with constant entries equal to $z_{0}$.) If $\mathcal{H}_{0}$ has infinite dimension, then the above condition for $\left\langle z, e_{\alpha}\right\rangle$, combined with the fact that $z$ must be a vector of finite length implies that $z=0$. In other words, $\left.\mathcal{U}\right|_{\mathcal{H}_{0}}$ does not admit a non-zero fixed vector in this case. 
- We now assume that $\rho \notin\{0,1\}$.

In particular, we begin with the case where $\operatorname{dim} \mathcal{H}_{0}=m<\infty$ and $\rho=-\frac{1}{m}$.

It was shown in [3] (see Proposition 4.9 and Theorem 4.10) that if $\mathcal{P}_{m+1} \subseteq \mathbb{M}_{m+1}(\mathbb{C})$ is the group of permutation matrices and $\mathbf{1}=(1,1, \ldots, 1)^{t} \in \mathbb{C}^{m+1}$, then $\mathcal{G}:=\left.\mathcal{P}_{m+1}\right|_{\mathbf{1}^{\perp}}$ is an irreducible group for which $\Omega=\left\{1,-\frac{1}{m}\right\}$ is an admissible set. Of course, for such an irreducible group, no (non-zero) fixed vector can exist.

Having said this, the proof of Theorem 4.10 of [3] shows that there exist an admissible vector $\xi$ corresponding to $\Omega$ and a basis $\left\{u_{1}, u_{2}, \ldots, u_{m}\right\}$ for $\mathbf{1}^{\perp} \subseteq \mathbb{C}^{m+1}$ so that

(i) with $u_{m+1}=-\sum_{j=1}^{m} u_{j}$, we have that

$$
\left\{u_{1}, u_{2}, \ldots, u_{m+1}\right\}
$$

has the CIP $-\rho$ property for $\rho=-\frac{1}{m}$;

(ii) $\mathcal{G} u_{k}=\left\{u_{1}, u_{2}, \ldots, u_{m+1}\right\}$ for any $1 \leq k \leq m$. We may assume without loss of generality that $u_{1}=\xi$.

Clearly every $U \in \mathcal{G}$ permutes the elements of $\mathcal{G} \xi$. Let

$$
\mathcal{U}=\left\{V \in \mathcal{G}: V u_{m+1}=u_{m+1}\right\} .
$$

By construction, $u_{m+1}$ is a fixed vector for $\mathcal{U}$. Moreover, $I \in \mathcal{U}$ implies that $1 \in\{\langle V \xi, \xi\rangle$ : $V \in \mathcal{U}\}$, while the existence of $W \in \mathcal{U}$ so that $W \xi=u_{2}$ implies that $\langle W \xi, \xi\rangle=\rho=-\frac{1}{m}$.

These two constructions show that in the case where $\operatorname{dim} \mathcal{H}_{0}=m<\infty$ and $\rho=-\frac{1}{m}$, fixed vectors may or may not exist.

- So we have reduced the problem to the case where $\rho \notin\{0,1\}$ and either

(i) $\operatorname{dim} \mathcal{H}_{0}=m$ and $\rho \neq-\frac{1}{m}$; or

(ii) $\operatorname{dim} \mathcal{H}_{0}=\infty$.

Suppose that (i) holds and choose $U_{1}, U_{2}, \ldots, U_{m} \in \mathcal{U}$ so that $\mathcal{C}:=\left\{U_{i} \xi: 1 \leq i \leq m\right\}$ is a basis for $\mathcal{H}_{0}$. In particular, these vectors are linearly independent and hence distinct.

Note that if $V \in \mathcal{U}$, then

$$
\left\langle U_{i} \xi, V \xi\right\rangle=\left\langle V^{*} U_{i} \xi, \xi\right\rangle \in \Omega .
$$

If $V \xi \notin\left\{U_{i} \xi: 1 \leq i \leq m\right\}$, then this forces

$$
\left\langle U_{i} \xi, V \xi\right\rangle=\rho, \quad 1 \leq i \leq m .
$$

Similarly, the fact that the vectors $U_{i} \xi, 1 \leq i \leq m$ are distinct implies that

$$
\left\langle U_{i} \xi, U_{j} \xi\right\rangle=\rho, \quad 1 \leq i \neq j \leq m .
$$

But $\rho \neq-\frac{1}{m}$, and so the results of Section 2 imply that there can be at most $m$ vectors in $\mathcal{H}_{0}$ with the CIP $-\rho$ property, a contradiction. This shows that $V \xi \in\left\{U_{i} \xi: 1 \leq i \leq m\right\}$ for all $V \in \mathcal{U}$.

Let

$$
z=U_{1} \xi+U_{2} \xi+\cdots+U_{m} \xi
$$

We claim that $z$ is a fixed point for $\mathcal{U}$. Note that for every $V \in \mathcal{U}$, the vectors $\left\{V U_{i} \xi: 1 \leq\right.$ $i \leq n\}$ must be distinct and thus give a re-ordering of $\left\{U_{i} \xi: 1 \leq i \leq n\right\}$. So

$$
V z=\sum_{i=1}^{n} V U_{i} \xi=\sum_{i=1}^{n} U_{i} \xi=z .
$$


Next, assume that $\mathcal{H}_{0}$ is infinite-dimensional. Then $\rho$ must be positive. An argument similar to that above shows that the set $\mathcal{U} \xi:=\{\mathcal{U} \xi: \mathcal{U} \in \mathcal{U}\}$ forms a maximal set with the CIP $-\rho$ property, and so by Theorem 2.12 , it must be of the form $\Gamma\left(\mathcal{E}_{\infty}, \rho\right)$ or $\Gamma\left(\mathcal{E}_{\infty}, \mathcal{F}, \rho\right)$. Let us allow for the case where $\mathcal{F}=\varnothing$, and adopt the notation $\Gamma\left(\mathcal{E}_{\infty}, \mathcal{F}, \rho\right)$ to handle both of these situations simultaneously.

Let $y$ be the vector constructed in Section 2.10 (where the set

$$
\Gamma\left(\mathcal{E}_{\infty}, \mathcal{F}, \rho\right)=\Gamma\left(\mathcal{E}_{\infty}, \rho\right) \cup\left\{g_{\lambda}:=y+\sqrt{1-\rho} f_{\lambda}\right\}_{\lambda \in \Lambda}
$$

is the maximal family in $\mathcal{H}_{0}$ with the CIP $-\rho$ property as shown in Proposition 2.11). Recall that $\langle y, W \xi\rangle=\rho$ for all $W \in \mathcal{U}$.

We claim that setting $z=y$ yields the desired fixed vector for $\mathcal{U}$. To show this, first note that for every $V \in \mathcal{U}$, we have that $V$ permutes the elements of $\mathcal{U} \xi=\Gamma\left(\mathcal{E}_{\infty}, \mathcal{F}, \rho\right)$, by virtue of the fact that it is bijective.

But then

$$
\langle y, V \xi\rangle=\left\langle y, x_{n}\right\rangle=\rho
$$

for some $n \geq 1$, where $\Gamma\left(\mathcal{E}_{\infty}, \rho\right)=\left\{x_{n}\right\}_{n=1}^{\infty}$, or (recalling that $f_{\lambda}$ is orthogonal to $y$ ) there exists $\lambda \in \Lambda$ such that

$$
\langle y, V \xi\rangle=\left\langle y, y+\sqrt{1-\rho} f_{\lambda}\right\rangle=\rho .
$$

Thus for every $U \in \mathcal{U}$,

$$
\langle U y-y, V \xi\rangle=\left\langle y, U^{*} V \xi\right\rangle-\langle y, V \xi\rangle=\rho-\rho=0
$$

for all $V \in \mathcal{U}$. Since $\{V \xi: V \in \mathcal{U}\}$ densely spans $\mathcal{H}_{0}$, we find that $U y-y=0$.

In both cases (a) and (b) of the proposition above, the restriction of $\mathcal{U}$ to $\mathcal{H}_{0}$ can be irreducible, as we have seen before.

4.2. Proposition. Let $\mathcal{H}$ be a complex Hilbert space and $\mathcal{U} \subseteq \mathcal{B}(\mathcal{H})$ be a group of unitary operators on $\mathcal{H}$. Suppose furthermore that $\Omega=\{\rho, 1\}$ is a two-element admissible set for $\mathcal{U}$, that $\xi \in \mathcal{H}$ is a corresponding admissible vector, and that $\rho=\langle W \xi$, xi $\rangle$ for some $W \in \mathcal{U}$. Set $\mathcal{H}_{0}=\overline{\operatorname{span}}\{U \xi: U \in \mathcal{U}\}$.

(a) If $\operatorname{dim} \mathcal{H}_{0}=: m<\infty$, then either

(i) $\rho \in\left\{-\frac{1}{m},-\frac{1}{m-1}\right\}$, or

(ii) $\left.\mathcal{U}\right|_{\mathcal{H}_{0}}$ is unitarily similar to a group of permutation matrices.

(b) If $\operatorname{dim} \mathcal{H}_{0}=\infty$, then there exists a subspace $\mathcal{M}_{0} \subseteq \mathcal{H}_{0}$ of codimension at most one such that $\left.\mathcal{U}\right|_{\mathcal{M}_{0}}$ is unitarily similar to a group of permutation unitaries

Proof. It is clear that $V \in \mathcal{U}$ implies that $\|V\| \leq 1$, and thus $|\rho| \leq 1$. Furthermore, $\rho=\langle W \xi, \xi\rangle$ implies that $\bar{\rho}=\left\langle W^{*} \xi, \xi\right\rangle \in \Omega$, forcing $\rho \in \mathbb{R}$. Since $|\Omega|=2$, this implies that $-1 \leq \rho<1$.

(a) Suppose that $\operatorname{dim} \mathcal{H}_{0}=: m<\infty$, and that $\rho \notin\left\{-\frac{1}{m},-\frac{1}{m-1}\right\}$.

By Theorem 4.1 and its proof, we see that there exist $U_{1}, U_{2}, \ldots, U_{m} \in \mathcal{U}$ such that

(I) $\mathcal{H}_{0}=\operatorname{span}\left\{U_{1} \xi, U_{2} \xi, \ldots, U_{m} \xi\right\}$,

(II) $0 \neq z:=U_{1} \xi+U_{2} \xi+\cdots+U_{m} \xi$ satisfies $U z=z$ for all $U \in \mathcal{U}$, and

(III) $V \xi \in\left\{U_{1} \xi, U_{2} \xi, \ldots, U_{m} \xi\right\}$ for all $V \in \mathcal{U}$. 
Note that

$$
\left\langle z, U_{j} \xi\right\rangle=1+(m-1) \rho
$$

for al $1 \leq j \leq m$, and that

$$
\|z\|^{2}=m[1+(m-1) \rho] \geq 0 .
$$

(This last estimate shows that $[1+(m-1) \rho] \geq 0$, which we shall use below.)

For $\lambda \in \mathbb{R} \backslash\{0\}$, and $1 \leq i \leq m$, consider

$$
\xi_{\lambda, i}:=\lambda U_{i} \xi-z \in \mathcal{H}_{0}
$$

Observe that for $1 \leq i \neq j \leq m$,

$$
\left\langle\xi_{\lambda, i}, \xi_{\lambda, j}\right\rangle=\rho \lambda^{2}-2[1+(m-1) \rho] \lambda+m[1+(m-1) \rho] .
$$

The polynomial $p(\lambda)=\rho \lambda^{2}-2[1+(m-1) \rho] \lambda+m[1+(m-1) \rho]$ always has a real root, by virtue of the fact that its discriminant, namely $2(1-\rho)[1+(m-1) \rho]$, is always non-negative. (Indeed, $-1 \leq \rho<1$ and $[1+(m-1) \rho] \geq 0$, as noted above.) Furthermore, that root can not be 0 , as $\rho \neq-\frac{1}{m-1}$.

Let $\lambda_{0}$ denote such a real root for the polynomial $p$. Then $\left\{\xi_{\lambda, i}: 1 \leq i \leq m\right\}$ is an orthogonal set in the $m$-dimensional space $\mathcal{H}_{0}$, and so

$$
\mathcal{B}_{0}:=\left\{\frac{\xi_{\lambda_{0}, i}}{\left\|\xi_{\lambda_{0}, i}\right\|}: 1 \leq i \leq m\right\}
$$

is an orthonormal basis for $\mathcal{H}_{0}$. It is then a simple consequence of (II) and (III) above that $V \xi_{\lambda_{0}, i} \in \mathcal{B}_{0}$ for all $V \in \mathcal{U}$. The fact that each $V \in \mathcal{U}$ is bijective then shows that $\mathcal{U}$ acts as a group of permutation matrices on $\mathcal{H}_{0}$ relative to the orthonormal basis $\mathcal{H}_{0}$.

(b) Now suppose that $\operatorname{dim} \mathcal{H}_{0}=\infty$.

In Theorem 4.1, we saw that if $\rho=0$, then $\left.\mathcal{U}\right|_{\mathcal{H}_{0}}$ is unitarily similar to a group of permutation unitaries.

Suppose therefore that $\rho \neq 0$.

Again, by Theorem 4.1, there exists a non-zero vector $z \in \mathcal{H}_{0}$ satisfying

(IV) $V z=z$ for all $V \in \mathcal{U}$,

(V) $\|z\|^{2}=\rho$, and

(VI) $\langle z, V \xi\rangle=\rho$ for all $V \in \mathcal{U}$.

For each $U \in \mathcal{U}$, set $\xi_{U}=U \xi-z \neq 0$. If $U, V \in \mathcal{U}$ and $U \xi \neq V \xi$, then a quick computation shows that $\left\langle\xi_{U}, \xi_{V}\right\rangle=0$, and thus $\mathcal{B}:=\left\{\frac{\xi_{U}}{\left\|\xi_{U}\right\|}: U \in \mathcal{U}\right\}$ is an orthonormal set.

1.

Let $\mathcal{M}_{0}:=\overline{\operatorname{span}} \mathcal{B} \subseteq \mathcal{H}_{0}$. It is clear that $\overline{\operatorname{span}}\{\mathcal{B}, z\}=\mathcal{H}_{0}$, so that $\operatorname{dim}\left(\mathcal{H}_{0} / \mathcal{M}_{0}\right) \leq$

Furthermore, each $V \in \mathcal{U}$ is unitary, hence bijective, and clearly $V$ acts as a permutation on the space $\mathcal{M}_{0}$ relative to the orthonormal basis $\mathcal{B}_{0}$.

\section{NONSELFADJOINT SEMIGROUPS OF OPERATORS}

5.1. In the previous section we completely characterized those two-element subsets $\Omega \subseteq \mathbb{C}$ which can occur as admissible sets for selfadjoint, irreducible semigroups $\mathcal{S}$ of operators acting on an infinite-dimensional, separable Hilbert space. 
If one removes the condition that the semigroup $\mathcal{S}$ be selfadjoint, the problem becomes significantly more complicated. In this section we pursue two separate lines of investigation. Firstly, for irreducible, unital semigroups of unitary operators, we obtain constraints on the nature of the possible two-element admissible sets, by showing that for any such admissible set $\Omega=\{\rho, 1\}$, we must have that $-1 \leq \rho<1$. Secondly, we show that any two-element subset $\Omega=\{\rho, 1\}$ of $\mathbb{C}$ can appear as an admissible set of some unital semigroup of operators, although that semigroup may be reducible.

In fact, depending upon the choice of $\rho$, we can infer the existence of fixed points for $\mathcal{G}$ (see Proposition 5.6).

5.2. Proposition. Suppose that $\mathcal{H}$ is an n-dimensional Hilbert space and that $\mathcal{S} \subseteq \mathcal{B}(\mathcal{H})$ is an irreducible semigroup of unitary operators. If $\Omega$ is a two-element admissible set for $\mathcal{S}$, then $\Omega=\left\{-\frac{1}{n}, 1\right\}$. Furthermore, for any $n \geq 2$, there exists an irreducible group $\mathcal{G} \subseteq \mathcal{B}\left(\mathbb{C}^{n}\right)$ for which $\left\{-\frac{1}{n}, 1\right\}$ is an admissible set.

Proof. Let $U \in \mathcal{S}$ be a unitary operator. Since $\operatorname{dim} \mathcal{H}=n<\infty, U$ is diagonalizable. It is an easy exercise to check that $U^{*}$ lies in the norm-closure $\overline{\mathcal{S}}$ of $\mathcal{S}$, and that $\Omega$ is an admissible set for $\overline{\mathcal{S}}$. Thus

- $U^{*} U=I \in \overline{\mathcal{S}}$, implying that $1 \in \Omega$; and

- $\overline{\mathcal{S}}$ is (easily seen to be) a group, which is irreducible since $\mathcal{S}$ was assumed to be irreducible.

The result now follows from Proposition 4.9 and Theorem 4.10 of [3].

5.3. Proposition. Let $\mathcal{H}$ be an infinite-dimensional, separable Hilbert space. Let $I \in \mathcal{S} \subseteq \mathcal{B}(\mathcal{H})$ be an irreducible, unital semigroup of unitary operators, and let $\xi$ be a unit vector in $\mathcal{H}$. Suppose that $\Omega:=\{\langle U \xi, \xi\rangle: U \in \mathcal{S}\} \subseteq \mathbb{C}$ is an admissible subset for $\mathcal{S}$, with $|\Omega|=2$. Then $\Omega=\{\rho, 1\}$ for some real number $-1<\rho<1$.

Proof. Since $I \in \mathcal{S}$, it is clear that $\Omega=\{\rho, 1\}$ for some $1 \neq \rho \in \mathbb{C}$. Furthermore, $S \in \mathcal{S}$ implies that $\|S\|=1$, whence $|\rho| \leq 1$. If $|\rho|=1$, then the fact that every element of $\mathcal{S}$ is unitary forces $\mathbb{C} \xi$ to be an invariant subspace for $\mathcal{S}$, contradicting the irreducibility of $\mathcal{S}$. Hence $|\rho|<1$.

We argue by contradiction. Suppose that $\rho \notin \mathbb{R}$. We claim that $\mathcal{K}:=\{S \in \mathcal{S}:\langle S \xi, \xi\rangle=$ $\rho\}$ is a semigroup ideal of $\mathcal{S}$.

Let $\varphi_{\xi}$ denote the state $\varphi_{\xi}(T)=\langle T \xi, \xi\rangle, T \in \mathcal{B}(\mathcal{H})$. Since $\mathcal{S}$ is an irreducible semigroup of $\mathcal{B}(\mathcal{H})$, we know that the restriction of $\varphi_{\xi}$ to $\mathcal{S}$ can not be constant. Thus $\mathcal{K}$ is not empty. Fix $S \in \mathcal{K}$, and extend the linearly independent set $\{\xi\}$ in $\mathcal{H}$ to an orthonormal basis so that the matrix for $S$ with respect to that basis is of the form:

$$
[S]=\left[\begin{array}{cccc}
\rho & s_{12} & s_{13} & \cdots \\
\left(1-|\rho|^{2}\right)^{\frac{1}{2}} & s_{22} & s_{23} & \cdots \\
0 & s_{32} & s_{33} & \cdots \\
\vdots & & &
\end{array}\right]
$$

If $T \in \mathcal{S}$, then with respect to this basis, either

$$
[T]=\left[\begin{array}{ccccc}
1 & 0 & 0 & \cdots & \\
0 & t_{22} & t_{23} & t_{24} & \\
0 & t_{32} & t_{33} & t_{34} & \cdots
\end{array}\right],
$$


or

$$
[T]=\left[\begin{array}{cccc}
\rho & t_{12} & t_{13} & \cdots \\
t_{21} & t_{22} & t_{23} & \cdots \\
t_{31} & t_{32} & t_{33} & \cdots \\
\vdots & & &
\end{array}\right] .
$$

In the first case, it is easy to check that $\langle T S \xi, \xi\rangle=\langle S \xi, \xi\rangle=\rho=\langle S T \xi, \xi\rangle$, so that $T S, S T \in \mathcal{K}$. In the second case, we find from the equation $\langle T S \xi, \xi\rangle \in \Omega$ that $\rho^{2}+t_{12} \sqrt{1-|\rho|^{2}} \in\{1, \rho\}$. Suppose that $\langle T S \xi, \xi\rangle=1$; i.e., that $\rho^{2}+t_{12} \sqrt{1-|\rho|^{2}}=1$. Then

$$
t_{12}=\frac{1-\rho^{2}}{\sqrt{1-|\rho|^{2}}},
$$

and so $\left|t_{12}\right| \geq \sqrt{1-|\rho|^{2}}$. But the first row of $T$ has norm at most 1 , from which we see that $\left|t_{12}\right| \leq \sqrt{1-|\rho|^{2}}$. In other words, $\left|t_{12}\right|=\sqrt{1-|\rho|^{2}}$. But then $\left|1-\rho^{2}\right|=\left.|1-| \rho\right|^{2} \mid$, from which it follows that $\rho \in \mathbb{R}$, contradicting our assumption. Thus $\langle T S \xi, \xi\rangle=1$; i.e. TS $\in \mathcal{K}$.

Next, suppose that $\langle S T \xi, \xi\rangle=1$. Then $\left\langle T \xi, S^{*} \xi\right\rangle=1$; but $T \xi$ is the first column of $T$, while $S^{*} \xi$ is the adjoint of the first row of $S$. Since each of these has norm at most one, the only way that their inner product can be 1 is if the first column of $T$ equals the adjoint of the first row of $S$. But then this forces $\rho=\bar{\rho}$, which in turn forces $\rho \in \mathbb{R}$, contradicting our hypothesis. Thus $S T \in \mathcal{K}$ as well.

Finally, $\mathcal{K}$ is a semigroup ideal. Since $\mathcal{S}$ is irreducible, so is $\mathcal{K}$. However, Lemma 3.2 shows that $\varphi_{\xi}(\mathcal{K})$ can not be constant, contradicting the definition of $\mathcal{K}$. This contradiction shows that $\rho \in \mathbb{R}$.

Next, we turn our attention to the question of which two-element subsets $\Omega$ of $\mathbb{C}$ appear as an admissible set for some unital semigroup of operators. We emphasize that we no longer require that the semigroup be irreducible. The existence of the identity operator in our semigroup obviously requires that 1 belong to $\Omega$.

5.4. Proposition. Let $\mathcal{H}$ be an infinite-dimensional, separable Hilbert space and $\rho \in \mathbb{C} \backslash\{1\}$. Then there exists a group $\mathcal{G} \in \mathcal{B}(\mathcal{H})$ for which $\Omega=\{\rho, 1\}$ is an admissible set.

Proof. The case $\rho=0$ is easily handled by selecting an orthonormal basis $\left\{e_{n}\right\}_{n=1}^{\infty}$ for $\mathcal{H}$ and considering the group $\mathcal{G}$ of all permutation unitary operators relative to this basis. (That is, $G \in \mathcal{G}$ if and only if there exists a permutation $\varphi: \mathbb{N} \rightarrow \mathbb{N}$ such that $G e_{n}=e_{\varphi(n)}$ for all $n \geq 1$. In this case, for any $n \geq 1$, the vector $\xi=e_{n}$ is an admissible vector corresponding to the admissible set $\Omega=\{0,1\}$.

If $\rho=-\frac{1}{n}$ for some $n \geq 1$, then by Proposition 4.9 of [3], there exists an (irreducible) group $\mathcal{R}_{n} \subseteq \mathbb{M}_{n}(\mathbb{C})$ for which $\Omega=\left\{-\frac{1}{n}, 1\right\}$ is an admissible set. If we then identify $\mathbb{M}_{n}(\mathbb{C})$ with $P_{n} \mathcal{B}(\mathcal{H}) P_{n}$ for some fixed projection $P_{n}$ of rank $n$, then we may set $\mathcal{G}=\left\{G \oplus\left(I-P_{n}\right)\right.$ : $\left.G \in \mathcal{R}_{n}\right\}$ to obtain the desired group.

Next, suppose that $\rho \in \mathbb{C}$, but $\rho \notin\{0,1\} \cup\left\{-\frac{1}{n}\right\}_{n=1}^{\infty}$.

For each $n \geq 1$, let $L_{n}=\left[\begin{array}{cc}\alpha_{n} & \beta_{n} \\ \beta_{n} & -\alpha_{n}\end{array}\right]$, where $\alpha_{n}:=\frac{\rho}{1+(n-1) \rho}$, and where $\beta_{n} \in \mathbb{C}$ is chosen so that $\alpha_{n}^{2}+\beta_{n}^{2}=1$. (There are two choices for each $\beta_{n}$; either one will do for our purposes.) 
Fix an orthonormal basis $\left\{e_{n}\right\}_{n=1}^{\infty}$ for $\mathcal{H}$, and define the operators $J_{n} \in \mathcal{B}(\mathcal{H})$ relative to this basis so that

$$
J_{n}=I_{n-1} \oplus L_{n} \oplus I
$$

Observe that $J_{n}^{2}=I, n \geq 1$, and let $\mathcal{G}=\left\langle J_{n}: n \geq 1\right\rangle$ be the group generated by the set $\left\{J_{n}\right\}_{n=1}^{\infty}$.

Clearly $G \in \mathcal{G}$ implies that $G=I+F$ for some finite-rank operator $F$, as this is true for all $J_{n}$. Define $x_{1}=e_{1}$ and, for $n \geq 2$, set

$$
x_{n}=\sum_{i=1}^{n-1} \gamma_{i} e_{i}+\left(\beta_{1} \beta_{2} \cdots \beta_{n-1}\right) e_{n}
$$

where $\gamma_{i}=\beta_{1} \beta_{2} \cdots \beta_{i-1} \alpha_{i}$ for all $i \geq 1$.

CLAIM. For any $G \in \mathcal{G}, G e_{1} \in\left\{x_{j}\right\}_{j=1}^{\infty}$.

A moment's reflection should suffice to convince the reader that it suffices to prove that $J_{k} x_{m} \in\left\{x_{j}\right\}_{j=1}^{\infty}$ for all $k, m \geq 1$. We verify this by considering four separate cases.

- CASE 1. Suppose $k \geq m+1$.

Since $x_{m}$ is supported on $\left\{e_{1}, e_{2}, \ldots, e_{m}\right\}$ and $k \geq m+1$, it is easy to verify that $J_{k} x_{m}=x_{m} \in\left\{x_{j}\right\}_{j=1}^{\infty}$.

- CASE 2. Suppose $k=m$.

Then $J_{k} x_{m}=J_{m} x_{m}=\sum_{i=1}^{m} y_{i} e_{i}$, where

$$
\begin{aligned}
y_{i} & =\gamma_{i}, 1 \leq i \leq m-1 \\
y_{m} & =\alpha_{m} \beta_{1} \beta_{2} \cdots \beta_{m-1} \\
y_{m+1} & =\beta_{m} \beta_{1} \beta_{2} \cdots \beta_{m-1} .
\end{aligned}
$$

Thus $J_{m} x_{m}=x_{m+1} \in\left\{x_{j}\right\}_{j=1}^{\infty}$.

- CASE 3. Suppose $k=m-1$.

Then $J_{k} x_{m}=J_{m-1} x_{m}=\sum_{i=1}^{m} y_{i} e_{i}$, where

$$
\begin{aligned}
y_{i} & =\gamma_{i}, 1 \leq i \leq m-2 \\
y_{m-1} & =\alpha_{m-1}\left(\alpha_{m-1} \beta_{1} \beta_{2} \cdots \beta_{m-2}\right)+\beta_{m-1}\left(\beta_{1} \beta_{2} \cdots \beta_{m-1}\right) \\
& =\left(\beta_{1} \beta_{2} \cdots \beta_{m-2}\right)\left(\alpha_{m-1}^{2}+\beta_{m-1}^{2}\right) \\
& =\beta_{1} \beta_{2} \cdots \beta_{m-2} \\
y_{m} & =\beta_{m-1}\left(\alpha_{m-1} \beta_{1} \beta_{2} \cdots \beta_{m-2}\right)+\left(-\alpha_{m-1}\right)\left(\beta_{1} \beta_{2} \cdots \beta_{m-1}\right) \\
& =0 .
\end{aligned}
$$

Thus $J_{m-1} x_{m}=x_{m-1} \in\left\{x_{j}\right\}_{j=1}^{\infty}$. 
- CASE 4. Suppose $k<m-1$. Using the fact that $\alpha_{k+1}=\frac{\alpha_{k}}{1+\alpha_{k}}, k \geq 1$, we find that $J_{k} x_{m}=\sum_{i=1}^{m} y_{i} e_{i}$, where

$$
\begin{aligned}
y_{i} & =\gamma_{i}, \quad 1 \leq i \leq m, i \notin\{k, k+1\} \\
y_{k} & =\alpha_{k}\left(\beta_{1} \beta_{2} \cdots \beta_{k-1} \alpha_{k}\right)+\beta_{k}\left(\beta_{1} \beta_{2} \cdots \beta_{k} \alpha_{k+1}\right) \\
& =\left(\beta_{1} \beta_{2} \cdots \beta_{k-1}\right)\left(\alpha_{k}^{2}+\alpha_{k+1} \beta_{k}^{2}\right) \\
& =\left(\beta_{1} \beta_{2} \cdots \beta_{k-1}\right)\left(\alpha_{k}^{2}+\frac{\alpha_{k}}{1+\alpha_{k}}\left(1-\alpha_{k}^{2}\right)\right) \\
& =\beta_{1} \beta_{2} \cdots \beta_{k-1} \alpha_{k} \\
y_{k+1} & =\beta_{k}\left(\alpha_{k} \beta_{1} \beta_{2} \cdots \beta_{k-1}\right)+\left(-\alpha_{k}\right)\left(\beta_{1} \beta_{2} \cdots \beta_{k} \alpha_{k+1}\right) \\
& =\left(\beta_{1} \beta_{2} \cdots \beta_{k}\right)\left(\alpha_{k}-\alpha_{k} \alpha_{k+1}\right) \\
& =\left(\beta_{1} \beta_{2} \cdots \beta_{k}\right)\left(\alpha_{k+1}\right),
\end{aligned}
$$

so that $J_{k} x_{m}=x_{m} \in\left\{x_{j}\right\}_{j=1}^{\infty}$.

This proves the claim.

Letting $\xi=x_{1}=e_{1}$ shows that $\langle G \xi, \xi\rangle \in\left\{1, \alpha_{1}\right\}=\{1, \rho\}$ for all $G \in \mathcal{G}$, completing the proof.

\subsection{Remark.}

1. We claim that $x_{\infty}:=\sum_{n=1}^{\infty} \gamma_{n} e_{n} \in \mathcal{H}$; i.e., that $\left(\gamma_{n}\right)_{n=1}^{\infty} \in \ell_{2}$.

To see this, note that for $n \geq 1, \alpha_{n}=\frac{\rho}{1+(n-1) \rho}$, from which it easily follows that $\left(\alpha_{n}\right)_{n=1}^{\infty} \in \ell_{2}$. It suffices, therefore, to show that the sequence $\left(\beta_{1} \beta_{2} \cdots \beta_{n-1}\right)_{n=1}^{\infty}$ is bounded. But, for $n \geq 1$,

$$
\begin{aligned}
&\left(\beta_{1} \beta_{2} \cdots \beta_{n-1}\right)^{2}=\left(1-\alpha_{1}^{2}\right)\left(1-\alpha_{2}^{2}\right) \cdots\left(1-\alpha_{n-1}^{2}\right) \\
&=\left(1-\alpha_{1}\right)\left[\left(1+\alpha_{1}\right)\left(1-\alpha_{2}\right)\right] \cdots\left[\left(1+\alpha_{n-2}\right)\left(1-\alpha_{n-1}\right)\right]\left(1+\alpha_{n-1}\right) \\
&=\left(1-\alpha_{1}\right)\left(1+\alpha_{n-1}\right), \\
& \text { as }\left(1+\alpha_{k}\right)\left(1-\alpha_{k+1}\right)=\left(1+\alpha_{k}\right)\left(1-\frac{\alpha_{k}}{1+\alpha_{k}}\right)=1,1 \leq k \leq n-2 .
\end{aligned}
$$

Since $\lim _{n \rightarrow \infty} \alpha_{n-1}=0$, we clearly see that $\sup _{n \geq 1}\left|\beta_{1} \beta_{2} \cdots \beta_{n-1}\right|<\infty$, as required.

2. The computation from Case 3 of the previous Proposition now shows that $J_{k} x_{\infty}=$ $x_{\infty}$ for all $k \geq 1$, so that $x_{\infty}$ is a fixed point for $\mathcal{G}$.

The existence of a fixed point for the group $\mathcal{G}$ from Proposition 5.4 above is not purely coincidental, as we now demonstrate. 
5.6. Proposition. Suppose that $\mathcal{H}$ is a complex, separable Hilbert space and that $\mathcal{G} \subseteq \mathcal{B}(\mathcal{H})$ is a bounded group. Suppose furthermore that $\rho \in \mathbb{C}$, but $\rho \notin(-\infty, 0] \cup\{1\}$. If $\Omega=\{\rho, 1\}$ is an admissible set for $\mathcal{G}$, then $\mathcal{G}$ has a fixed point. That is, there exists $w \in \mathcal{H}$ such that $G w=w$ for all $G \in \mathcal{G}$.

Proof. Let $\xi$ be a norm-one admissible vector corresponding to $\Omega$, and consider the (bounded) set $\mathcal{C}:=\{G \xi: G \in \mathcal{G}\}$. The boundedness of $\mathcal{C}$ implies that the weak closure $\overline{\mathcal{C}}^{w}$ of $\mathcal{C}$ is compact. If $x$ is any element of the $\overline{\mathcal{C}}^{w}$ in $\mathcal{H}$, then the fact that $\langle G \xi, \xi\rangle \in \Omega$ for all $G \in \mathcal{G}$ implies that $\langle x, \xi\rangle \in \Omega$, and thus $x \neq 0$.

Next, let $K=\operatorname{co}\left(\overline{\mathcal{C}}^{w}\right)$, so that $K$ is a weakly compact, convex subset of $\mathcal{H}$. It is relatively straightforward to check that $z \in K$ implies that $\langle z, \xi\rangle \in c o(\{\rho, 1\})$, the line segment from $\rho$ to 1 . The condition we have imposed upon $\rho$ ensures that $0 \notin c o(\{\rho, 1\})$, and hence that $z \in K$ implies that $z \neq 0$.

Also, the fact that $\mathcal{G}$ is a semigroup ensures that $G K \subseteq K$ for all $G \in \mathcal{G}$, and the fact that $\mathcal{G}$ is a bounded set of linear operators implies that $\mathcal{G}$ acts equicontinuously upon $K$. By Kakutani's Fixed Point Theorem (see [1], p. 457), there exists $w \in K$ such that $G w=w$ for all $G \in \mathcal{G}$.

\section{REFERENCES}

[1] N. Dunford and J.T. Schwartz. Linear operators. Part I. General theory. John Wiley and Sons, Inc., New York, 1958.

[2] J. Jedwab and A. Wiebe. Constructions of complex equiangular lines from mutually unbiassed bases. arXiv1408.5169v2[math.CO], 20 March, 2015.

[3] L.W. Marcoux, M. Omladič, A.I. Popov, H. Radjavi, and B.R. Yahaghi. Ranges of vector states on irreducible operator semigroups. Semigroup Forum, DOI 10.1007/s00233-015-9772-7, 2016.

[4] H. Radjavi and P. Rosenthal. Simultaneous Triangularization. Universitext. Springer Verlag, New York, Berlin, Heidelberg, 2000.

[5] G. Zauner. Quantendesigns, Grundzüge einer nichtkommutativen Designtheorie. PhD thesis, University of Vienna, 1999.

Department of Pure Mathematics, University of Waterloo, Waterloo, Ontario, Canada N2L 3G1

E-mail address: LWMarcoux@uwaterloo.ca

Department of Pure Mathematics, University of Waterloo, Waterloo, Ontario, Canada N2L 3G1

E-mail address: hradjavi@uwaterloo.ca

Department of Mathematics, Faculty of Sciences, Golestan University, Gorgan 4913815759, IRAN

E-mail address: bamdad5@hotmail.com 